\title{
Direct Numerical Simulation of a high-pressure turbulent reacting temporal mixing layer
}

\author{
Josette Bellan ${ }^{\mathrm{a}, \mathrm{b}, *}$

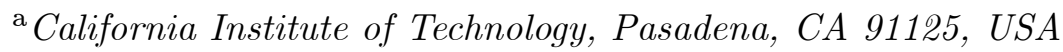 \\ ${ }^{\mathrm{b}}$ Jet Propulsion Laboratory, California Institute of Technology, Pasadena, CA \\ 91109, USA
}

\begin{abstract}
Direct Numerical Simulation realizations were created of a temporal mixing layer in which combustion occurs under high-pressure (high- $p$ ) turbulent conditions. The model combines the formulation of [1] for describing multi-species mixing under high- $p$ conditions and a single-step chemical reaction of rate consistent with ignition prediction [2]. In each simulation the computations are pursued past a time at which a maximum average-volumetric $p$ is attained; most analysis is performed at this time, $t_{p p}^{*}$. The ensemble of realizations explores the effect of the initial Reynolds number, $\mathrm{Re}_{0}$, of the initial pressure, $p_{0}$, and of the initial composition of the two mixing-layer streams. The results show that the thermodynamic energy added by the reaction at the small scales is partially dissipated and partially backscattered. The formation of turbulent small scales is initiated by the morphological changes in the flow through stretching and twisting rather than vice versa. The reaction establishes primarily in the oxidizer stream and is preponderantly of diffusion type. Overwhelmingly, the higher reaction rates occur in the diffusion flame, particularly in regions of high density-gradient magnitude. At higher $p_{0}$ the reaction rate reaches higher values and occurs in regions of higher density gradients. The range of reaction rates is independent of the $\mathrm{Re}_{0}$ value but the magnitude of the density gradients increases with $\mathrm{Re}_{0}$. When $\mathrm{H}_{2} \mathrm{O}$ and $\mathrm{CO}_{2}$ are initially present, uphill diffusion dominates over regular diffusion and occurs in regions of smaller density-gradient magnitude whereas regular diffusion occurs in regions of larger density-gradient magnitude where the reaction is more vigorous. $\mathrm{H}_{2} \mathrm{O}$ is more prone than $\mathrm{CO}_{2}$ to regular diffusion in the larger density-gradient magnitude regions. When $\mathrm{H}_{2} \mathrm{O}$ and $\mathrm{CO}_{2}$ only form in the flame, both $\mathrm{H}_{2} \mathrm{O}$ and $\mathrm{CO}_{2}$ are subject to regular diffusion over the entire range of density-gradient values. The dissipation probability density function is a log normal distribution at large dissipation values.
\end{abstract}

Key words: high-pressure combustion; uphill diffusion during high-pressure combustion; flame index at high pressure; Direct Numerical Simulation

Preprint submitted to Combustion and Flame

25 September 2016

(C) 2016. This manuscript version is made available under the Elsevier user license http://www.elsevier.com/open-access/userlicense/1.0/ 


\section{Introduction}

High-pressure (high- $p$ ) reactive flows are encountered in numerous propulsion devices such as Diesel, HCCI, gas turbine and liquid rocket propulsion engines. Also, cooling management is one of the crucial issues in advanced gas turbine and scramjet engines and it is usually addressed by injecting high- $p$ endothermic fuel through the pipes; the fuel acts as a heat exchanger and absorbs the heat of the pipe walls, a fact which promotes its chemical decomposition [3]. What characterizes all reactions under these situations is the fact that $p$ is larger than the critical pressure, $p_{c}$ (subscript $c$ denotes the critical state), of the reactants. In engines, the reactions occur under fully turbulent conditions whereas in the heat pipes the Reynolds number (Re) generally does not exceed 10,000. Despite the important applications of the topic of high- $p$ (i.e. $p>40$ bar) turbulent reactive flows, there is scant understanding of the coupling among high- $p$ conditions (involving thermodynamics and transport properties), turbulence (a dynamic characteristic of the flow) and chemical reactions. Large Eddy Simulations (LESs) have been performed for turbulent combustion at $p$ larger than atmospheric, but still within the range of validity of the perfect gas assumption [4]. Current advanced simulations of high- $p$ reactive flows are either of LES type (e.g. $[5,6,7])$ in which models for small-scale processes and turbulent combustion must be used, or of laminar type (e.g. [8]) in which the emphasis is on accurate transport properties and detailed kinetics. Between these two types of simulations there is a gap: Direct Numerical Simulation (DNS) of high- $p$ turbulent combustion with accurate transport properties can reveal the coupling among high- $p$, turbulence and reaction rate to help gain a better understanding of high- $p$ combustion and eventually lead to more appropriate turbulent reaction models. Despite the relatively smaller Re values achievable in DNS compared to applications, because in DNS one resolves all scales overwhelmingly responsible for the dissipation, it is well known that DNS results can be a powerful tool in turbulence research [9]. The goal of this study is to unravel some of the salient aspects of these high- $p$ turbulent reactive flows through DNS.

From the previous investigation of high- $p$ turbulent mixing among several species [1] it is evident that the advanced modeling of the fluxes [10] and of transport properties in fully-populated matrix form [11,12] is crucial in predicting the mixture composition prior to ignition. That study was conducted within the framework of DNS of a temporal mixing layer wherein the more abundant mass fraction species were initially segregated whereas trace species could be initially either segregated or present in both streams of the mixing layer; during turbulent mixing, one of the most detectable feature of these

\footnotetext{
* Corresponding author. Tel: (818) 354-6959, Fax: (818) 393-6682.

Email address: Josette.Bellan@jpl.nasa.gov (Josette Bellan).
} 
flows is the presence of high density-gradient magnitude (HDGM) regions. Concurrently, the process of uphill diffusion, which is of molecular diffusion origin (unlike the well-known atmospheric- $p$ counter-gradient turbulent diffusion that is of convective origin), was identified that segregates species into regions of low and high concentration even when a species is initially uniformly distributed in the flow. This segregation is the result of inter-species diffusion and creates species gradients which act akin to a solid mesh and induce turbulence in the flow. Therefore, flows experiencing uphill diffusion become more turbulent but in turn turbulence has the effect of suppressing uphill diffusion [13] and thus the ultimate result is difficult to predict without specific simulations. Experimental data from binary-species mixing discussed in [14] showed that observations obtained under fully turbulent conditions qualitatively matched DNS pivotal findings from similar studies at smaller Re values, thereby emphasizing the appropriateness of temporal mixing layer DNS studies for understanding fully-turbulent spatial jet flows.

A limited DNS study of high- $p$ turbulent reacting flow [2] showed that these uphill-diffusion/turbulence aspects prevail during reaction and that the flame is of a partially premixed type. Because of the brief nature of that reactive-flow study, many aspects of high- $p$ turbulent reacting flow remain unknown. Since the fuel and oxidizer are initially segregated, ignition must be promoted by diffusion $[15,16]$ which occurs primarily in the HDGM regions. But how does the specific mixture becomes established in the HDGM regions? Do these HDGM regions prevail during combustion (since turbulence tends to homogenize a flow)? What are the roles played by the diffusion flame and by the premixed flame in consuming the fuel?

This study explores the unknown aspects stated above with the goal of deriving a fundamental understanding of the physical processes at play and of their coupling; the primary interest is in the physics of the cold-ignition regime. The governing equations are briefly listed in section 2 (for simplicity the multiple-species transport property models are relegated to Appendix A) and the choice of the reaction mechanism and rate is presented in section 3. The configuration, boundary conditions and initial conditions are described in section 4. The numerical method for solving the equations using DNS is briefly explained in section 5. A database is created consisting of four DNS realizations. The results are presented in section 6 and address the complex physics of high- $p$ turbulent reactive flow. After discussing the evolution of the flow in section 6.1 , in section 6.2 evidence is presented to confirm that transitional states have been achieved for each simulation prior to reaction initiation. The visualizations of section 6.3 show that even at higher initial Reynolds number, $\mathrm{Re}_{0}$, than in the previous study [2] the flame is of partially-premixed type. In section 6.4, visualizations of the HDGM regions are presented showing that these regions do exist during combustion; given the HDGM importance, an examination is performed of the relationship between reaction rate and dif- 
fusion flame or premixed flame, between reaction rate and density gradient magnitude and between composition and diffusion in the HDGM regions. The results highlight the ability of the diffusion model to discriminate according to molecular size and molar mass between the seemingly similar aspects of the trace species diffusion. The dissipation probability density function (PDF) in the presence of uphill diffusion is also examined. A summary and conclusions discuss the salient findings in section 7 .

\section{Governing equations}

\subsection{Differential conservation equations}

The conservation equations have been derived in detail elsewhere $[1,17]$ and are here briefly stated

$$
\begin{gathered}
\frac{\partial \rho}{\partial t}+\frac{\partial}{\partial x_{j}}\left[\rho u_{j}\right]=0 \\
\frac{\partial}{\partial t}\left(\rho u_{i}\right)+\frac{\partial}{\partial x_{j}}\left[\rho u_{i} u_{j}+p \delta_{i j}-\sigma_{i j}\right]=0 \\
\frac{\partial}{\partial t}\left(\rho e_{t}\right)+\frac{\partial}{\partial x_{j}}\left[\left(\rho e_{t}+p\right) u_{j}-u_{i} \sigma_{i j}+q_{j}\right]=0 \\
\frac{\partial}{\partial t}\left(\rho Y_{\alpha}\right)+\frac{\partial}{\partial x_{j}}\left[\rho Y_{\alpha} u_{j}+J_{\alpha j}\right]=m_{\alpha} \dot{\omega}_{\alpha}, \alpha \in[1, N-1],
\end{gathered}
$$

where $t$ denotes the time, subscripts $i$ and $j$ refer to the components of spatial coordinate $\mathbf{x}, \rho$ is the density, $u_{i}$ is the $i$-th velocity component, $e_{t}=e+\frac{1}{2} u_{i} u_{i}$ is the total energy where $e$ is the internal energy, $Y_{\alpha}$ is the mass fraction of species $\alpha, m_{\alpha}$ is the $\alpha$-th species molar mass, $N$ is the total number of species, $\sigma_{i j}$ is the Newtonian viscous stress tensor

$$
\sigma_{i j}=\mu\left(2 S_{i j}-\frac{2}{3} S_{k k} \delta_{i j}\right), \quad S_{i j}=\frac{1}{2}\left(\frac{\partial u_{i}}{\partial x_{j}}+\frac{\partial u_{j}}{\partial x_{i}}\right)
$$

where $S_{i j}$ is the strain rate tensor, $\mu$ is the viscosity, $J_{\alpha j}$ and $q_{j}$ are respectively the $\alpha$-th species mass flux and the heat flux in the $j$-th direction and $\dot{\omega}_{\alpha}$ is the $\alpha$-th species reaction rate. The expressions for the species mass fluxes and

the heat flux use the full matrices of mass-diffusion coefficients and thermal- 
diffusion factors $[11,18,19,20]$

$$
\begin{aligned}
J_{\alpha j} & =-\rho\left[Y_{\alpha}\left(D_{T, \alpha}\right) \frac{1}{T} \frac{\partial T}{\partial x_{j}}+Y_{\alpha}\left(D_{p, \alpha}\right) \frac{1}{p} \frac{\partial p}{\partial x_{j}}+\sum_{\beta=1}^{N-1}\left(D_{\alpha \beta}^{\prime} \frac{m_{\alpha}}{m_{\beta}}\right) \frac{\partial Y_{\beta}}{\partial x_{j}}\right] \\
q_{j} & =-\lambda \frac{\partial T}{\partial x_{j}}+\sum_{\alpha=1}^{N-1} J_{\alpha j} A_{\alpha}
\end{aligned}
$$

where $T$ is the temperature and

$$
A_{\alpha}=\left[\left(\frac{h_{\alpha}}{m_{\alpha}}-\frac{h_{N}}{m_{N}}\right)-R_{u} T\left(\frac{\bar{\alpha}_{T, \alpha}^{b}}{m_{\alpha}}-\frac{\bar{\alpha}_{T, N}^{b}}{m_{N}}\right)\right] .
$$

Since $\sum_{\alpha=1}^{N} Y_{\alpha}=1$, the consideration of the set of $(N-1)$ species equations rather than the set of $N$ dependent species ensures that the equations are independent; in these $(N-1)$ equations, the original fluxes were rewritten to account for only $(N-1)$ gradients. In Eqs. (6) - (8)

$$
\begin{gathered}
D_{T, \alpha}=-\sum_{\beta=1}^{N} \bar{\alpha}_{T, \beta}^{b} \mathbb{D}_{\beta \alpha}, \quad D_{p, \alpha}=\frac{p}{R_{u} T} \sum_{\beta=1}^{N} v_{\beta} \mathbb{D}_{\beta \alpha}, \quad D_{\alpha \gamma}=\sum_{\beta=1}^{N} \mathbb{D}_{\alpha \beta} \alpha_{D \beta \gamma}, \\
\bar{\alpha}_{T, \alpha}^{b}=\sum_{\beta=1}^{N} X_{\beta} \alpha_{T, \beta \alpha}^{b}, \quad D_{\alpha \beta}^{\prime}=D_{\alpha \beta}-\left(1-\frac{m_{\beta}}{m_{N}}\right)\left(\sum_{\gamma=1}^{N-1} D_{\alpha \gamma} X_{\gamma}\right) .
\end{gathered}
$$

Here, $X_{\alpha}=Y_{\alpha} m / m_{\alpha}$ represents the molar fraction; $m$ is the mixture molar mass, $m=\sum_{\gamma=1}^{N} m_{\gamma} X_{\gamma} ; v_{\alpha}=\left(\partial v / \partial X_{\alpha}\right)_{T, p, X_{\beta}(\beta \neq \alpha)}$ is the partial molar volume, where the molar volume is $v=m / \rho ; h_{\alpha}=\left(\partial h / \partial X_{\alpha}\right)_{T, p, X_{\beta}(\beta \neq \alpha)}$ is the partial molar enthalpy, where the molar enthalpy is $h=G-T(\partial G / \partial T)_{p, X}$ with $G$ being the Gibbs energy; $R_{u}$ is the universal gas constant; $D_{\alpha \gamma}$ are the pairwise mass diffusion coefficients; and $\alpha_{T, \alpha \beta}^{b}$ are the binary thermal diffusion factors. The mass-diffusion factors, $\alpha_{D \alpha \beta}$, are calculated from thermodynamics as

$$
\begin{aligned}
\alpha_{D \alpha \beta} & \equiv \frac{1}{R_{u} T} X_{\alpha} \frac{\partial \mu_{\alpha}}{\partial X_{\beta}}=\left(\delta_{\alpha \beta}-\delta_{\alpha N}\right)+X_{\alpha}\left(R_{\alpha \beta}-R_{\alpha N}\right), \quad 1 \leqslant \alpha \leqslant N, \\
R_{\alpha \beta} & \equiv \frac{\partial \ln \gamma_{\alpha}}{\partial X_{\beta}}, \quad 1 \leqslant \alpha \leqslant N, 1 \leqslant \beta \leqslant N
\end{aligned}
$$

where $\mu_{\alpha}$ is the chemical potential of species $\alpha$ written in terms of $(N-1)$ species; $\gamma_{\alpha} \equiv \varphi_{\alpha} / \varphi_{\alpha}^{o}$, where $\varphi$ is the fugacity coefficient written in terms of $N$ species and the superscript $o$ denotes the pure $\left(X_{\alpha}=1\right)$ limit. Matrix elements $\mathbb{D}_{\beta \gamma}$ are the solution of the mixing rules equations $[11]$

$$
\sum_{\beta=1}^{N}\left[\delta_{\alpha \beta}-\left(1-\delta_{\alpha \beta}\right) X_{\beta} \frac{\overline{\mathcal{D}}_{\alpha}}{\mathcal{D}_{\alpha \beta}^{b}}\right] \frac{\mathbb{D}_{\beta \gamma}}{X_{\beta}}=\overline{\mathcal{D}}_{\alpha} \frac{\left(\delta_{\alpha \gamma}-Y_{\alpha}\right)}{X_{\alpha}},
$$




$$
\overline{\mathcal{D}}_{\alpha}=1 / \sum_{\substack{\beta=1 \\ \beta \neq \alpha}}^{N}\left(\frac{X_{\beta}}{\mathcal{D}_{\alpha \beta}^{b}}\right) .
$$

Solutions for $\mathbb{D}_{\beta \gamma}$ may be obtained by an approximate inversion [21] yielding

$$
\begin{aligned}
\mathbb{D}_{\beta \gamma} & \simeq X_{\beta} \mathbb{D}_{\beta \gamma}^{(1)} \\
\mathbb{D}_{\alpha \beta}^{(1)} & =\frac{\left(1+Y_{\alpha}\right)}{X_{\alpha}} \mathcal{D}_{\alpha}^{*} \delta_{\alpha \beta}+\left(1-\delta_{\alpha \beta}\right) \frac{\mathcal{D}_{\alpha}^{*} \mathcal{D}_{\beta}^{*}}{\mathcal{D}_{\alpha \beta}^{b}}-\left(\sigma_{\alpha} \mathcal{D}_{\alpha}^{*}+\sigma_{\beta} \mathcal{D}_{\beta}^{*}\right)+\sum_{\gamma=1}^{N}\left(Y_{\gamma} \sigma_{\gamma} \mathcal{D}_{\gamma}^{*}\right) \\
\mathcal{D}_{\alpha}^{*} & =\left(1-Y_{\alpha}\right) \overline{\mathcal{D}}_{\alpha}, \\
\sigma_{\alpha} & =\frac{m_{\alpha}}{m}\left(1+Y_{\alpha}\right)+\sum_{\substack{\beta=1 \\
\beta \neq \alpha}}^{N} Y_{\beta} \frac{\mathcal{D}_{\beta}^{*}}{\mathcal{D}_{\alpha \beta}^{b}}
\end{aligned}
$$

where $\mathcal{D}_{\alpha \beta}^{b}$ is the full approximation binary-diffusion matrix. This inter-species diffusion model, Eqs. (14) - (18) is not valid when the mixture is composed of a single species (e.g. in pure fuel zones). In that case, Eq. (14) is no longer used and the diffusion coefficients are evaluated using the binary-diffusion matrix by setting $\mathcal{D}_{\alpha}^{*}=\mathcal{D}_{\alpha N}^{b}$, where $N$ represents the index associated with the solvent. This method was tested against an exact Gauss inversion and it gave the same results, with an additional gain in computational time. Defining $\mathcal{D}_{\alpha \beta}$ as the first approximation of the binary diffusion matrix and realizing that the deviation of the ratio $\mathcal{D}_{\alpha \beta}^{b} / \mathcal{D}_{\alpha \beta}$ from unity is comparable to uncertainties in binary diffusion coefficients values [11], we assume $\mathcal{D}_{\alpha \beta}^{b}=\mathcal{D}_{\alpha \beta}$. The computation of the mixture physical viscosity, $\mu_{p h}$, of the mixture physical thermal conductivity, of $\lambda_{p h}$, of $\mathcal{D}_{\alpha \gamma}$ and of $\alpha_{T, \alpha \gamma}^{b}$ is presented in Appendix A.

\subsection{The equation of state}

Equations (1)-(4) are coupled with the Peng-Robinson (PR) EOS

$$
p=\frac{R_{u} T}{\left(v_{P R}-b_{m i x}\right)}-\frac{a_{m i x}}{\left(v_{P R}^{2}+2 b_{m i x} v_{P R}-b_{m i x}^{2}\right)}
$$

from which $T$ and $p$ are obtained as an iterative solution which satisfies both values of $\rho$ and of $e$, as obtained from the conservation equations [22]. Here $v_{P R}$ is the molar PR volume, and $v=v_{P R}+v_{s}$ where $v_{s}$ is the volume shift introduced so as to improve the accuracy of the PR EOS at high $p[25] ; a_{\text {mix }}$ and $b_{m i x}$ are functions of $T$ and $X_{i}$ (see Appendix B). The $v_{s}$ computation in the context of DNS was explained in detail elsewhere [22]. 


\section{Reaction rate for ignition studies}

The fuel of interest is $\mathrm{C}_{7} \mathrm{H}_{16}$ because it is representative of the processes occurring during ignition in diesel combustion. Because DNS is a tool to understand the fundamental physics rather than perform a practical computation from which one would expect quantitative accuracy, a single-step reaction is considered here; single reaction models were used in the past in DNS of atmospheric combustion, e.g. [23,24]. Essentially, the DNS study will not be qualitatively sensitive to the number of reactions because using a single-step reaction retains the crucial aspects of (1) molecular transport among (rather than between) several species, and (2) interaction among chemistry, high- $p$ multi-species transport, high- $p$ thermodynamics and turbulence.

The simplest overall reaction representing the $\mathrm{C}_{7} \mathrm{H}_{16}$ oxidation is

$$
\mathrm{C}_{7} \mathrm{H}_{16}+11 \mathrm{O}_{2}+\mathrm{N}_{2} \rightarrow 7 \mathrm{CO}_{2}+8 \mathrm{H}_{2} \mathrm{O}+\mathrm{N}_{2}
$$

and its rate can be expressed as

$$
\dot{\omega}=A T^{n} \frac{\rho^{a+b}}{m_{\mathrm{C}_{7} \mathrm{H}_{16}} m_{\mathrm{O}_{2}}} Y_{\mathrm{C}_{7} \mathrm{H}_{16}}^{a} Y_{\mathrm{O}_{2}}^{b} \exp \left(-E_{a} / R_{u} T\right)
$$

where $E_{a}$ is the activation energy and $A$ is the pre-exponential constant. The ability of a single-step reaction mechanism to capture the laminar flame speed of a wide range of hydrocarbon/air flames was investigated in [26], and for n-heptane it was found that in the $[1,4]$ range of equivalence ratios the best fit compared to the experimental data was obtained for $a=0.25$, $b=1.5, E_{a}=30 \mathrm{kcal} / \mathrm{mol}, A=5.1 \times 10^{11} \mathrm{~cm}^{3}(\mathrm{~mol} \times \mathrm{s})^{-1}$ and $n=0$. However, the present study is devoted to ignition in a turbulent flow rather than to flame propagation in a laminar flow. When the reaction rate of Eq. (21) was here tested for ignition using the laminar flame kinetic parameters, the results indicated that the minimum ignition delay time always occurred for very lean mixtures, a fact which is contrary to the findings of other authors (e.g. [27]) who investigated n-heptane/air autoignition using either global [28,29] or reduced kinetic mechanisms [30] and always reported ignition to initiate in rich mixtures when the oxidizer $T$ is larger than the fuel $T$. This confirmed that the kinetic parameters proposed in [26] are not suitable for ignition computations, and thus a reaction rate expression with $a=b=1$ was used here to ensure that the minimum ignition delay time occurred in rich mixtures. As in [26], $E_{a}=30 \mathrm{kcal} / \mathrm{mol}$ and $n=0$, whereas $A=9.6 \times 10^{6} \mathrm{~cm}^{3}(\mathrm{~mol} \times \mathrm{s})^{-1}$ here to avoid abrupt ignition.

Since the interest is on combustion in air, the species in the mixture are $\mathrm{C}_{7} \mathrm{H}_{16}$, $\mathrm{O}_{2}, \mathrm{~N}_{2}, \mathrm{CO}_{2}$ and $\mathrm{H}_{2} \mathrm{O}$. Of note, with $a=b=1, \dot{\omega} \propto \rho^{2}$ and thus $\dot{\omega}$ strongly depends on $p$. 


\section{Configuration, boundary conditions and initial conditions}

The configuration is that of a temporal mixing layer with periodic boundary conditions in the streamwise $\left(x_{1}\right)$ and spanwise $\left(x_{3}\right)$ directions and nonreflecting boundary conditions in the cross-stream $\left(x_{2}\right)$ direction [31]. The domain size in the streamwise $\left(L_{1}\right)$ and spanwise $\left(L_{3}\right)$ directions is such that it accommodates initially four vortices associated with the wavelengths $\lambda_{1}$ and $\lambda_{3}$ of perturbations (see [1] for details), respectively. The cross-stream domain size $\left(L_{2}\right)$ is larger than in the study of [1] and is selected to ensure that there is no interference of the mixing region with the domain boundaries even after combustion significantly enlarges this mixing region.

\subsection{Transport properties for DNS computations}

By definition, $\operatorname{Re}_{0} \equiv\left[0.5\left(\rho_{U}+\rho_{L}\right) \Delta U_{0} \delta_{\omega, 0}\right] / \mu_{R}$ where $\rho_{U}$ and $\rho_{L}$ are mixture initial densities, with subscripts $U$ and $L$ labeling the upper and lower streams respectively; $\delta_{\omega, 0}=\Delta U_{0} /\left(\partial u_{0} / \partial x_{2}\right)_{\max }$ is the initial vorticity thickness computed by using $u_{0}$ which is the $\left(x_{1}, x_{3}\right)$ planar average of the initial velocity in the streamwise direction; $\Delta U_{0}=U_{U}-U_{L}$ is the initial velocity difference across the layer; and as already stated, $\mu_{R}$ is a reference viscosity. According to the principle of flow similarity [32], a flow's characteristics only depend on nondimensional numbers rather than individual transport properties. To ensure that all non-dimensional numbers are accurately computed, first a physical initial mixture viscosity $\mu_{p h, 0}$ is calculated based on the physical initial species viscosities (Appendix A.1), then a reference value $\mu_{R}$ is obtained from the chosen $\operatorname{Re}_{0}$ value, and finally a factor $\mathcal{F} \equiv \mu_{R} / \mu_{p h, 0}$ is defined. All transport properties computed during the simulation are then scaled by $\mathcal{F}$, thereby ensuring the computation of accurate dimensionless numbers (e.g. the Schmidt and Prandtl numbers, $S c$ and $\operatorname{Pr}$ ). The value of $\mathcal{F}$ was computed at $t=0$ for the initial constant $p_{0}$ and $T_{0}$, using a mixture composed of five species, each species mass fraction being averaged over the entire domain. Thus, a unique $\mathcal{F}$ value is employed in the computational domain; see Table 1.

\subsection{Initial profiles and reaction initiation}

The initial profiles are represented by mean quantities upon which perturbations are imposed. The mean profiles are error functions and the perturbations are found using an analytical solution (see Appendix B in [1]).

Complete initial conditions for all computations are listed in Table 1; in particular, $\lambda_{1} / \delta_{\omega, 0}=7.29$ and $\lambda_{3}=0.6 \lambda_{1}$ as in [33]. Computations with $\mathrm{Re}_{0}=2000$ 
required smaller values of amplitudes of the vorticity perturbations $F_{3 D}$ and $F_{2 D}$ (see definition in [1] and values in Table 1 caption) because otherwise the computation showed lack of convergence at very early stages. In all simulations $T_{U}=1000 \mathrm{~K}$ and $T_{L}=600 \mathrm{~K}$. Listed in Table 2 are the initial compositions of the free-streams for all simulations.

The properties of these five species are listed in Table 3. The interaction coefficients necessary to compute $p$ from the EOS for this set of species are provided in Table 4 (see Appendix B for notations and details); coefficients with unknown values are assumed null.

As reported by [1], each simulation reaches a transitional state denoted by the subscript $t r$ and the non-dimensional transitional time $t_{t r}^{*}$ is defined as that at which the one-dimensional fluctuation-based spectra for the velocities and the thermodynamic variables become smooth, except for the forcing frequency; $t^{*} \equiv t \Delta U_{0} / \delta_{\omega, 0}$. In each computation the reaction is initiated at $t_{t r}^{*}$ by activating the pre-exponential constant in Eq. (21). The value of the momentum-thickness-based Reynolds number, $\operatorname{Re}_{m} \equiv \operatorname{Re}_{0} \delta_{m} / \delta_{\omega, 0}$, at important time stations of the computation is also listed in Table 1; the momentum thickness, $\delta_{m}$, is defined by Eq. (22).

\section{$5 \quad$ Numerical method}

The differential equations of section 2.1 combined with the EOS described in section 2.2, using the transport coefficient models presented in Appendix A and the reaction rate of section 3, were numerically solved using a fourth-order explicit Runge-Kutta time integration and a sixth-order compact scheme spatial discretization. Following well-established techniques for turbulent reactive flows, operator splitting was used to efficiently solve the conservation equations. Time stability was achieved by filtering the conservative variables every five time steps using a tenth-order filter. Compared to [1], the implementation of the more accurate algorithm for computing $T$ and $p$ additionally allows the use of boundary filters (of the same type as the domain-core filter) which are now utilized at the $x_{2}$ boundary. According to [34], this filtering does not affect the physical content of the data because it only removes spurious information as it only acts on the shortest wavelengths that can be resolved on the grid; thus, the filter does not act as a turbulence model which would permit under-resolved computations.

The computations were parallelized using three-dimensional domain decomposition and message passing. The tridiagonal solver for the compact derivative scheme was efficiently parallelized using the method of [35]. 
The grid spacing, $\Delta x$, is uniform and is selected to ensure that the smallest scales relevant to dissipation are resolved and that there is no accumulation of energy at those scales (see section 6.2). The grid spacings used in various realizations are listed in Table 1. Based on the results of [1], the smallest scales are of thermodynamic origin, and according to [36] these thermodynamic scales also have more morphological complexity than the dynamic scales. Because there are no criteria to estimate beforehand the thermodynamic scale magnitude that would be similar to the criteria existing for estimating the Kolmogorov scale, a certain amount of experimentation and intuition based on the study of [1] were necessary to obtain a very good local and instantaneous representation of the smallest scales. The domain lengths, $L_{i}, i=1$ and 3 , are the same for all simulations while $L_{2}$ is simulation dependent.

\section{Results}

Compared to a previous brief investigation [2], the present database contains a simulation at an initial Reynolds number that is double of that previously used. Moreover, the present scope of the investigation is much wider: the focus is to understand the characteristics of the flow and compare them before and after ignition and to explore the PDF of the dissipation which plays a role in turbulent reaction-rate modeling. Since the mixing layers are inherently statistically unsteady, time-averaged results are not statistically significant in the present study. Additionally, it is well known that systems potentially containing several phases (as could be the case in the high- $p$ regime where thermodynamic fluctuations exist) are not ergodic [37] because one or several order parameters - i.e., additional variables - must be provided to describe the state of the system [38]; obtaining statistical results under these conditions would entail creating a large number of DNS similar to those listed in Table 1 and perform statistics over solutions from simulations having very small differences in initial conditions. Considering the very substantial computational time necessary to obtain each DNS solution, creating a large enough database for performing statistics is unfeasible. Therefore, the adopted strategy is here to focus on the commonalities of the results for all DNS listed in Table 1 and consider these common aspects as being representative of the situation of interest. This approach is consistent with experimental and theoretical information showing that the flows of present interest have distinct inhomogeneities $[1,17,22,39,40]$, in which case statistical averages, which smear these aspects, may not be representative of the local aspect of the flow since these local inhomogeneities affect turbulence production $[1,41]$ as well as phase separation $[38,42,43]$. These arguments lead to the analysis being focussed on the state obtained at a characteristic time $t_{p p}^{*}$ (defined in section 6.1) although earlier states are also examined if pertinent information can be extracted. 
The results are organized as follows: In section 6.1 the temporal evolution of the layer is described, in section 6.2 it is shown that the flow in which combustion is initiated has indeed turbulent characteristics, and in section 6.3 the thermodynamic fields at $t_{p p}^{*}$ are visualized, allowing the identification of the flame-type during combustion. Then, in section 6.4 the focus is on the reaction rate and the composition. Indeed, similar to the previous non-reactive binary-species [17] and to the multi-species mixing [1] studies, the flow develops HDGM regions in which mixing occurs. The goal is to understand the role of the HDGM regions in the reaction, and particularly how the composition develops there. The dissipation is addressed in section 6.5.

\subsection{Evolution of the global quantities}

The time evolution of the momentum thickness

$$
\delta_{m}=\frac{\int_{x_{2}, \min }^{x_{2}, \max }\left[\left\langle\rho u_{1}\right\rangle_{x_{2}, \max }-\left\langle\rho u_{1}\right\rangle\right]\left[\left\langle\rho u_{1}\right\rangle-\left\langle\rho u_{1}\right\rangle_{x_{2}, \min }\right] d x_{2}}{\left(\left\langle\rho u_{1}\right\rangle_{x_{2}, \max }-\left\langle\rho u_{1}\right\rangle_{x_{2}, \min }\right)^{2}},
$$

non-dimensionalized as $\delta_{m} / \delta_{\omega, 0}$, where $x_{2, \min }=-L_{2} / 2.5, x_{2, \max }=x_{2, \min }+L_{2}$, and \langle\rangle symbolizes averages over homogeneous $\left(x_{1}, x_{3}\right)$ planes is illustrated in Fig. 1a. Other quantities displayed in Fig. 1 are the domain-averaged (symbol $\langle\langle\rangle\rangle)$ positive spanwise vorticity $\left\langle\left\langle\omega_{3}^{+}\right\rangle\right\rangle \delta_{\omega, 0} / \Delta U_{0}$, the domain-averaged enstrophy $\left\langle\left\langle\omega_{i} \omega_{i}\right\rangle\right\rangle\left(\delta_{\omega, 0} / \Delta U_{0}\right)^{2}$, and the domain-averaged relative pressure $\left(\langle\langle p\rangle\rangle-p_{0}\right)$; the figure arrangement is conducive to easy comparisons between layer growth and $p$ evolution, and between small-scale formation measured by $\left\langle\left\langle\omega_{3}^{+}\right\rangle\right\rangle \delta_{\omega, 0} / \Delta U_{0}$ and morphological evolution of the flow embodied in $\left\langle\left\langle\omega_{i} \omega_{i}\right\rangle\right\rangle\left(\delta_{\omega, 0} / \Delta U_{0}\right)^{2}$.

According to $\delta_{m} / \delta_{\omega, 0}$, all layers perturbed with the same amplitudes $F_{3 D}$ and $F_{2 D}$ grow similarly and the growth abates by $t_{t r}^{*}$ (at which time-station the spectra are smooth; see section 6.2); the imperceptibly thinner R1000p60a mixing layer is the manifestation of the lack of uphill diffusion during mixing $[1,2]$ resulting in reduced turbulent characteristics (see discussion below). Uphill diffusion, defined as existing when $\mathbf{J}_{\alpha} /\left(-\nabla Y_{\alpha}\right)<0$ [44], is a molecularinduced process due to diffusion occurring among (as opposed to between) species in a mixture and results in one or more species locally diffusing against its mass fraction gradient or its molar fraction gradient, leading to the formation of regions of high concentration of this species, so that effectively this species separates from the other species in the mixture $[44,45]$; the species gradients created through uphill diffusion act as a solid mesh would, producing turbulence [1]. Because of the reduced $F_{3 D}$ and $F_{2 D}$ used for R2000p60 (see Table 1), this layer starts growing later, eventually almost catches up in size with the other layers, peaks just after $t_{t r}^{*}$ and then begins a more pronounced 
decay. Once the reaction is initiated, the energy released is partially converted into dynamic energy, and all layers begin to grow again at a rate which is seemingly governed by the energy released.

As manifested by $\left\langle\left\langle\omega_{3}^{+}\right\rangle\right\rangle \delta_{\omega, 0} / \Delta U_{0}$, initially there is no dynamic small-scale formation, but as the spanwise vortices pair and the layer grows, small-scale formation drastically begins and increases, with a small abatement before the last vortex pairing. During mixing, the R1000p60a is slightly less turbulent than the other layers at the same pressure due to lack of uphill diffusion $[1,2]$, but after ignition its rate of decay is similar to the other $\operatorname{Re}_{0}=1000$ layers. During the early evolution, the R1000p80 layer experiences an imperceptibly reduced small scale formation due to the denser fluid in the layer that is more difficult to entrain. All $\mathrm{Re}_{0}=1000$ layers exhibit small subsequentlyoccurring secondary peaks which correspond to the turbulence created by the energy released during reaction. In concert with the discussion for $\delta_{m} / \delta_{\omega, 0}$, the small-scale formation for R2000p60 is initiated later than for the other layers but occurs at a faster rate and eventually surpasses in magnitude by almost a factor of two those of the $\mathrm{Re}_{0}=1000$ layers; the same qualitative features of small abatement before the ultimate vortex pairing and subsidiary small peak indicative of the effect of the reaction-energy released on small-scale formation are present, although this peak is considerably reduced in magnitude compared to the $\mathrm{Re}_{0}=1000$ layers. The vorticity in these reaction-induced small scales decays, presumably due to dissipation and also due to backscatter which transmits energy to the larger scales and thus supports the enhanced layer growth past ignition (see Fig. 1a), as all layers grow unabated.

According to $\left\langle\left\langle\omega_{i} \omega_{i}\right\rangle\right\rangle\left(\delta_{\omega, 0} / \Delta U_{0}\right)^{2}$, the morphological complexity of the flow resulting from twisting and stretching begins concurrently with small scale formation. However, the peak enstrophy occurs well before that of the spanwise vorticity thus indicating that the former is partially responsible for the latter. Increasing $\mathrm{Re}_{0}$ by a factor of two results in approximately a doubling of the peak enstrophy, whereas increasing $p_{0}$ by a third slightly reduces $\left\langle\left\langle\omega_{i} \omega_{i}\right\rangle\right\rangle\left(\delta_{\omega, 0} / \Delta U_{0}\right)^{2}$ due to the difficulty of entraining a denser fluid. As discussed in $[1,2]$, when $\mathrm{CO}_{2}$ and $\mathrm{H}_{2} \mathrm{O}$ are initially absent, the lack of uphill diffusion during mixing also reduces $\left\langle\left\langle\omega_{i} \omega_{i}\right\rangle\right\rangle\left(\delta_{\omega, 0} / \Delta U_{0}\right)^{2}$. Compared to the $\left\langle\left\langle\omega_{i} \omega_{i}\right\rangle\right\rangle\left(\delta_{\omega, 0} / \Delta U_{0}\right)^{2}$ mean values, the reaction seems to play a considerably more subdued role in morphologically enhancing the flow than in producing dynamic small scales. The conjecture is that this aspect is due to the fact that the reaction energy is released locally and as such it directly influences small-scale formation whereas morphological aspects of the flow are governed by gradients and thus are not local.

Starting from the initial condition, $\left(\langle\langle p\rangle\rangle-p_{0}\right)$ decreases (consistently with a reduction in $\langle\langle T\rangle\rangle$; see [2]), but as ignition occurs there is an abrupt growth and a culmination point is eventually reached at a time denoted by $t_{p p}^{*}$; 
this culmination point either corresponds to the subsidiary peaks seen in $\left\langle\left\langle\omega_{3}^{+}\right\rangle\right\rangle \delta_{\omega, 0} / \Delta U_{0}$ or occurs thereafter and thus the conjecture is that it is indicative of ignition and combustion. Since the reaction rate is higher when $\mathrm{CO}_{2}$ and $\mathrm{H}_{2} \mathrm{O}$ are not initially present (because there are initially more reactants), the peak is larger for R1000p60a than for R1000p60 and the temporal gradient of $\left(\langle\langle p\rangle\rangle-p_{0}\right)$ is higher. At larger $\operatorname{Re}_{0}$, the $\left(\langle\langle p\rangle\rangle-p_{0}\right)$ increase is delayed, this being attributed to both the more turbulent flow and the thinner mixing layer (see Fig. 1a) the effects of which combine to reduce the probability of $\mathrm{C}_{7} \mathrm{H}_{16}$ and $\mathrm{O}_{2}$ mixing at the molecular level to initiate reaction (i.e. stirring compared to molecular mixing); this effect also reduces the $\left(\langle\langle p\rangle\rangle-p_{0}\right)$ temporal gradient compared to the other layers having the same $p_{0}$, and results in the ultimate peak being slightly smaller than that of all $p_{0}=60$ atm simulations. At $p_{0}=80 \mathrm{~atm},\left(\langle\langle p\rangle\rangle-p_{0}\right)$ increases more sharply than at $p_{0}=60 \mathrm{~atm}$ due to the strong dependence of $\dot{\omega}$ on $\rho$ and thus on $p$, and reaches a value approximately $60 \%$ greater than for the $p_{0}=60$ atm simulations.

As an indication of the turbulence level achieved, the values of $\operatorname{Re}_{m}$ at $t_{t r}^{*}$ and $t_{p p}^{*}$ are provided in Table 1 indicating that $\mathrm{Re}_{m}$ increases between those time stations by approximately a 26\% for R1000p60 and R1000p60a, by 25\% for R1000p 80 and by $8 \%$ for R2000p60; thus this increase is clearly governed by $F_{3 D}$ and $F_{2 D}$ and only very slightly dependent on $p_{0}$.

\subsection{One-dimensional spectra at transition}

Figure 2 illustrates the spanwise and streamwise spectra, $E(k)$, of both dynamic and thermodynamic variables for all simulations. The spectra of the thermodynamic variables are shown because for high- $p$ flows often $\operatorname{Pr}>1$ $[1,46,47], S c_{\alpha}$ can be larger than unity [1] and iso-surfaces of the dissipation have much more morphological complexity than those representing dynamics [36], all of which show that the thermodynamic scales are smaller than the dynamic scales. Clearly, all spectra are smooth (except for the small peak at the perturbation frequency) as typically seen in turbulent flows, and they show excellent resolution by having no energy accumulation at the smallest scales. As expected, R2000p60 has spectra extending over a wider range of scales than the simulations having $\mathrm{Re}_{0}=1000$. Increasing $p_{0}$ from 60 atm to 80 atm only minimally extends the spectra to smaller scales. In all evidence, the reaction is initiated in a flow with turbulent characteristics. 


\subsection{Visualizations of the temperature, mass fractions and flame index}

The Flame Index [48] is typically defined as $G_{F O} \equiv \nabla Y_{\mathrm{C}_{7} \mathrm{H}_{16}} \cdot \nabla Y_{\mathrm{O}_{2}}$ and has been used to differentiate between diffusion flames $\left(G_{F O}<0\right)$ and premixed flames $\left(G_{F O}>0\right)$, a fact which makes it a natural diagnostic to understand the reaction burning mode. However, species are transported by the fluxes rather than by gradients, and thus the existing definition can only be applied to either Fickian diffusion or to situations in which the diagonal approximation of the diffusion matrix is used. Since in the present situation there is a complete Stefan-Maxwell diffusion matrix, the definition of the Flame Index is revised:

$$
G_{F O} \equiv \mathbf{J}_{\mathrm{C}_{7} \mathrm{H}_{16}} \cdot \mathbf{J}_{\mathrm{O}_{2}} .
$$

Illustrated in Fig. 3 are $T, G_{F O}$ and the species mass fractions in the $x_{3} / L_{3}=$ $1 / 16$ plane at $t_{p p}^{*}$ for R2000p60. For the purpose of this evaluation, $G_{F O}$ was considered a relevant quantity only in regions where $T>1150 \mathrm{~K}$, that is, where there is a flame; the results were insensitive to this threshold value providing it was chosen close to the value of $T_{U}$. According to the results depicted in Fig. 3, the flame overwhelmingly resides in the upper stream where the oxidizer resided initially. The highest $T$ values are in several diffusion flames and these flames are relatively thick compared to the global reactive region; since a single-step reaction underestimates in extent the reactive region, the implication is that for more complex kinetic mechanisms, the diffusion flame would be even visually thicker because there would be more species interdiffusing. These diffusion flames are bordered by premixed flames, and in the lower stream the burning is uniquely in a premixed mode. Since diffusion is slower under high- $p$ conditions [1] and since the slowest time always governs in any given process, the thick diffusion flames are a manifestation of the limiting factor for the reaction to proceed.

The reactants distribution shows that the flame burned almost all the fuel which diffused into the upper stream and all of the $\mathrm{O}_{2}$ available that did not diffuse elsewhere; this is reasonable since the flame was limited by the amount of diffused fuel rather than by the total amount of available $\mathrm{O}_{2} \cdot \mathrm{O}_{2}$ is still present in the central part of the mixing layer where fuel is also available, but $T$ may not be large enough there to initiate the reaction. The conjecture is that the possible negative effective thermal conductivity identified by [1] (the effective thermal conductivity may be negative in regions of uphill species mass diffusion) may be responsible for the reduced heat transfer ahead of the flame. Clearly, intense mixing occurs in the lower stream where the initial fuel boundary has receded. This mixing process ahead of the reaction zone is discussed in more detail in section 6.4.3.

The product species, $\mathrm{CO}_{2}$ and $\mathrm{H}_{2} \mathrm{O}$, are formed in the flame regions and their largest mass fraction magnitude exceeds by one order of magnitude their initial 
value which was uniform over the entire domain.

The present results are entirely relevant to fully-turbulent high- $p$ reactive flows because the present model is based on the same dynamic, thermodynamic and transport property mathematical formulation which compared qualitatively well with experimental evidence from several sources that was assembled in [14], showing that at $\operatorname{Re} \sim 6 \times 10^{5}$ and high- $p$ conditions there is evidence of strong density gradients, and thus of intense molecular mixing.

\subsection{Reaction and composition characteristics of the mixing layer}

\subsubsection{Reaction in the premixed and diffusion flames at $t_{p p}^{*}$}

Since the visualizations of Fig. 3 show that the reaction is of the partially premixed type, it is of interest to understand the distribution of the reaction in the diffusion and premixed flames. Displayed in Fig. 4a is $\dot{\omega}$ conditioned on $G_{F O, n}=G_{F O} / \max _{V}\left|G_{F O}\right|$ where $V=L_{1} \times L_{2} \times L_{3}$. The plotted $\dot{\omega}$ is restricted to values larger than $\dot{\omega}_{\max _{V}} \times 10^{-2} \mathrm{~mol} /\left(\mathrm{m}^{3} \mathrm{~s}\right)$ and represents averages over all points in $V$ for each of the $G_{F O} / \max _{V}\left|G_{F O}\right|$ values, meaning that it is the conditional average. For diffusion flames, it is clear that the reaction occurs over a wide range of $G_{F O, n}$ values, showing that it is qualitatively insensitive to the alignment of $\mathbf{J}_{\mathrm{C}_{7} \mathrm{H}_{16}}$ and $\mathbf{J}_{\mathrm{O}_{2}}$; the opposite is true for the premixed flame, as expected, where the alignment of the fluxes is unimportant since fuel and oxidizer are present in sufficient amounts for burning to occur. The results show that the overwhelming amount of reaction occurs for $G_{F O, n}<0$, that is, in diffusion flames, and the maximum conditional-average $\dot{\omega}$ occurs for $G_{F O, n}=-1$, indicating the beneficial effect of the fluxes of fuel and oxidizer being counter aligned, i.e. directed in-line toward each other, for maximizing the reaction. For premixed flames, the conditional-average $\dot{\omega}$ has generally a smaller (for R1000p60 and R1000p60a) or negligible (for R1000p80 and R2000p60) value. Therefore, the limiting factor for the reaction to occur is the ensemble of processes in the regions of high diffusion, that is, where the density gradient is large: the HDGM regions. The analysis of these processes is provided in section 6.4.3.

Because $\max _{V}\left|G_{F O}\right|$ may have different values for each of the simulations listed in Table 1 the conditional reaction rate values are not comparable among simulations in Fig. 4a; the only valid comparison is among slopes. The slope of the conditional $\dot{\omega}$ in the $G_{F O, n}<0$ regime indicates that the range of $\dot{\omega}$ values is considerably larger for R1000p80 and R1000p60 than for the R1000p60a and R2000p60, a fact which is conjectured to result from the more intense burning in the latter two cases (see section 6.4.3 discussing Fig. 4b). 


\subsubsection{HDGM regions at $t_{p p}^{*}$}

In Fig. 5, visualizations of $|\nabla \rho|$ are displayed at $t_{p p}^{*}$; in each figure, the yellow line indicates the stoichiometric locus. According to expectation, Figs. 3a and $5 \mathrm{~d}$ show that the largest $T$ occurs at the stoichiometric locus. As Fig. 3 already shows, the primary region of large diffusion has switched from the upper and lower boundary of the mixing layer during species mixing (see figure 15 of [1]) to be now overwhelmingly at the lower boundary of the layer where intense mixing occurs; since mixing is here concentrated in narrower regions, the maximum representative values of $|\nabla \rho|$ are here more than twice those encountered during mixing alone. It is this mixing in the HDGM regions which forms the pockets with equivalence ratio conducive to ignition, and thus the processes within the HDGM regions are crucial for reaction continuation.

With increasing $\mathrm{Re}_{0}$, the morphology of these HDGM regions becomes more complex due to the more turbulent flow, whereas with increasing $p_{0}$ the morphological complexity of these regions decreases due to the reduced turbulence level resulting from the difficulty to entrain a denser fluid. The absence of initial $\mathrm{CO}_{2}$ and $\mathrm{H}_{2} \mathrm{O}$ (i.e. in R1000p60a) does not appear to influence the morphological complexities of the HDGM regions at $t_{p p}^{*}$.

\subsubsection{Reaction inside the HDGM regions}

The above analysis highlights the importance of the diffusion flame. Clearly, the HDGM regions are the site of the diffusion processes leading to the diffusion flame. To learn about the relationship between $|\nabla \rho|$ and $\dot{\omega}$, conditional averages of $|\nabla \rho|$ are presented in Fig. 4b. Evidently, the higher is the reaction rate, the higher is the conditional $|\nabla \rho|$, meaning that the sites of the higher reaction rate are in the HDGM regions having larger $|\nabla \rho|$ values. The implication is that the availability of $\mathrm{C}_{7} \mathrm{H}_{16}$ and $\mathrm{O}_{2}$ in the HDGM regions favors the reaction and that the inter-species diffusion has brought the reactive species together in closer stoichiometric proportion in the regions with the larger $|\nabla \rho|$ (according to Fig. 3a, the $T$ values do not play a role as important as the composition). This observation motivates the examination in section 6.4.4 of the evolution of the composition in the HDGM regions.

According to Fig. 4b, the range of reaction-rate magnitudes in the HDGM regions is very much $p_{0}$ dependent as well as initial-composition dependent, in concert with Eq. (21), increasing both with $p_{0}$ (R1000p80) and with the increasing availability of reactants (R1000p60a); but this range is not influenced by the value of $\mathrm{Re}_{0}$. Rather, the value of $\mathrm{Re}_{0}$ (compare $\mathrm{R} 2000 \mathrm{p} 60$ with R1000p60) influences the magnitude of $|\nabla \rho|$ at specified $\dot{\omega}$ - a larger $\operatorname{Re}_{0}$ induces a larger $|\nabla \rho|$ at $t_{p p}^{*}$ - this being the manifestation of the shorter characteristic time available for mixing at the larger $\operatorname{Re}_{0}$. At specified $\dot{\omega}$, larger 
$|\nabla \rho|$ is also obtained for the larger $p_{0}$ due to the difficulty of entraining a denser fluid.

\subsubsection{Composition of the HDGM regions}

Since the largest values of $\dot{\omega}$ are encountered in the largest magnitude $|\nabla \rho|$ regions, it is intriguing to find out the evolution of the composition in the HDGM regions. To this end, the conditional averages of $Y_{\alpha}$ on $|\nabla \rho|$ are illustrated in Figs. 6(a,c,e,g) at $t^{*}=30$ which is an early time station before transition to turbulence, and in Figs. 6(b,d,f,h) at the respective $t_{p p}^{*}$. In each case, the minimum $|\nabla \rho|$ was selected to be $0.5 \times 10^{5} \mathrm{~kg} / \mathrm{m}^{4}$ and the maximum, $|\nabla \rho|_{\max _{V}}$, was that found in $V$. At $t^{*}=30$, the smallest value of $|\nabla \rho|_{\max _{V}}$ is found for R2000p60, this being a manifestation of the smaller initial perturbation amplitudes, while the largest $|\nabla \rho|_{\max _{V}}$ is encountered for R1000p80 due to the smaller diffusivities at larger $p[1]$. All $|\nabla \rho|$ regions contain $\mathrm{C}_{7} \mathrm{H}_{16}$, $\mathrm{O}_{2}, \mathrm{CO}_{2}$ and $\mathrm{H}_{2} \mathrm{O}$, except for the R1000p60a simulation in which the last two species are initially absent. For each simulation, as $|\nabla \rho|$ increases, so generally does $Y_{C_{7} H_{16}}$ while $Y_{O_{2}}$ decreases, except for R1000p60 where a small reversal occurs at the larger $|\nabla \rho|$. For the simulations initiated with $\mathrm{CO}_{2}$ and $\mathrm{H}_{2} \mathrm{O}$ present, both $Y_{\mathrm{H}_{2} \mathrm{O}}$ and $Y_{\mathrm{CO}_{2}}$ are almost uniformly distributed in the HDGM regions at their constant initial value, with an imperceptible decline toward the larger- $|\nabla \rho|$ regions. Extrapolating to $|\nabla \rho|=0$, it appears that the HDGM regions are initiated in regions of relatively (with respect to the maximum) large amounts of oxidizer and relatively small amounts of fuel.

By $t_{p p}^{*}, Y_{C_{7} H_{16}}\left(Y_{O_{2}}\right)$ has increased (decreased) compared to its value at $t^{*}=30$ for every simulation, while still maintaining a positive (negative) gradient towards the larger- $|\nabla \rho|$ regions, however, the gradient is much smaller. The largest $|\nabla \rho|$ now occurs for R2000p60 (in contrast to $t^{*}=30$ ), consistent with the findings of Fig. 4b. Since according to Fig. $4 \mathrm{~b}$ the larger- $|\nabla \rho|$ regions correspond to those of higher reaction rate, the implication is that fresh fuel is preferentially entrained in the HDGM regions as a precursor to reaction; as the gateway to the larger- $|\nabla \rho|$ magnitude regions, the smaller- $|\nabla \rho|$ magnitude regions accumulate more fuel (compared to the $t^{*}=30$ situation) since once inside HDGM regions further penetration into the larger- $|\nabla \rho|$ regions preponderantly relies on diffusion rather than entrainment. Indeed, according to the experimental work of [41], the HDGM regions act akin to solid boundaries, and therefore entrainment into the HDGM regions is impeded. The observation that $Y_{\mathrm{O}_{2}}$ is smaller in the larger- $|\nabla \rho|$ regions at $t_{p p}^{*}$ than at $t^{*}=30$ means that, beyond diffusion, if there is a limiting factor concerning the diffusion flame in the larger- $|\nabla \rho|$ regions, it is the availability of $\mathrm{O}_{2}$ rather than $\mathrm{C}_{7} \mathrm{H}_{16}$. Combustion also increases imperceptibly $Y_{\mathrm{CO}_{2}}$ and $Y_{\mathrm{H}_{2} \mathrm{O}}$.

Analysis of the DNS database in [1] revealed that $\mathrm{H}_{2} \mathrm{O}$ was undergoing up- 
hill diffusion during mixing with the other four species, and that $\mathrm{CO}_{2}$ was subject to the same phenomenon. When $\mathrm{CO}_{2}$ and $\mathrm{H}_{2} \mathrm{O}$ were initially present only in the upper stream of the mixing layer, uphill diffusion did not occur at $t_{t r}^{*}$ while initially unequal amounts of each $\mathrm{CO}_{2}$ and $\mathrm{H}_{2} \mathrm{O}$ in the upper and lower streams did lead to uphill diffusion. Preliminary information from the study of [2] identified uphill diffusion occurring for $\mathrm{H}_{2} \mathrm{O}$ during combustion. To find out whether and/or how uphill diffusion depends on the initial conditions, we first consider homogeneous plane $\left(x_{1}, x_{3}\right)$ PDFs of the angle between $\nabla Y_{\alpha}$ and $\mathbf{J}_{\alpha}$ for $\mathrm{H}_{2} \mathrm{O}$ (Fig. 7) and $\mathrm{CO}_{2}$ (Fig. 8) at several values of $|\nabla \rho|$, computed both at $t^{*}=30$ and $t_{p p}^{*}$. Since $\mathbf{J}_{\alpha} /\left(-\nabla Y_{\alpha}\right)>0$ is indicative of regular diffusion whereas $\mathbf{J}_{\alpha} /\left(-\nabla Y_{\alpha}\right)<0$ signifies uphill diffusion [44], this means that $\mathbf{J}_{\alpha} \cdot \nabla Y_{\alpha}<0$ corresponds to regular diffusion and $\mathbf{J}_{\alpha} \cdot \nabla Y_{\alpha}>0$ corresponds to uphill diffusion; the angle between $\mathbf{J}_{\alpha}$ and $\nabla Y_{\alpha}$ is thus in the ]$\pi / 2, \pi]$ range for regular diffusion and $[0, \pi / 2[$ range for uphill diffusion. For $\mathrm{H}_{2} \mathrm{O}$ (Fig. 7), the results show that at $t^{*}=30$ the PDF is quasi bimodal with a distinct segregation between the preponderant uphill diffusion and the secondary regular diffusion; only for R1000p60 and the largest- $|\nabla \rho|$ value is there a departure from the bimodal PDF, as secondary peaks populate mostly the ] $0, \pi / 2$ ] region. The largest uphill or regular diffusion probability is encountered at the smallest $|\nabla \rho|$ value, and the largest probability of finding either one is for R2000p60. By $t_{p p}^{*}$, the PDFs are still quasi bimodal but the regions of non-alignment (either positive or negative) are larger than at $t^{*}=30$ and the maximum probability of regular diffusion now occurs in the largest- $|\nabla \rho|$ rather than smallest- $|\nabla \rho|$ regions. For R1000p60, diffusion is still primarily of the uphill type, although the probability of regular diffusion at $\pi$ has substantially increased with respect to that at $t^{*}=30$. In the largest- $|\nabla \rho|$ regions, for both R1000p80 and R2000p60, at $t_{p p}^{*}$ the main mode of diffusion is of regular type and occurs near $\pi$. For R1000p 80 , in the intermediate- $|\nabla \rho|$ value regions, diffusion is most probable to be of uphill type with a very close-inmagnitude second-most-probable peak for the regular diffusion; whereas for the smallest- $|\nabla \rho|$ value regions the two types of alignment seem equally probable. The situation is similar for R2000p60 except that for the smallest- $|\nabla \rho|$ value, uphill diffusion remains the most probable. Clearly, what distinguishes R1000p80 and R2000p60 from R1000p60 is that for the former two simulations the most probable type of diffusion in the largest- $|\nabla \rho|$ value regions is of regular type. Based on the findings in [1] that uphill diffusion promotes the formation of small turbulent scales and the evidence in [13] that turbulence suppresses uphill diffusion, the findings of Fig. 6 showing that the maximum density gradient increases from $t^{*}=30$ to $t_{p p}^{*}$ for R1000p80 and R2000p60 but decreases for R1000p60 is attributed to the turbulent scales formed at the largest $|\nabla \rho|$ value seemingly dominating the competition between uphill diffusion and turbulence, and smearing the HDGM regions in that $|\nabla \rho|$ range.

In examining Fig. 8 showing the corresponding PDFs for $\mathrm{CO}_{2}$, we see a marked difference from the results of Fig. 7: the probability of regular diffusion is much 
smaller than that of uphill diffusion for all these simulations which initially contain $\mathrm{H}_{2} \mathrm{O}$ and $\mathrm{CO}_{2}$, and this occurs at all times and values of $|\nabla \rho|$ except for R1000p 80 for the largest- $|\nabla \rho|$ value regions. The difference between the patterns for $\mathrm{H}_{2} \mathrm{O}$ and $\mathrm{CO}_{2}$ is attributed to the different molecule size and $m_{\alpha}$ of these two species. At high- $p$ conditions, as $p$ is larger, $\rho$ increases due to the molecules being closer together and 'caging' may occur wherein molecules are trapped by surrounding molecules with the result that molecular diffusion diminishes. Since caging occurs in regions of higher $\rho$, it is expected that the locations most likely subject to it are those where relatively cold $\mathrm{C}_{7} \mathrm{H}_{16}$ is entrained; according to Fig. 6 , when $\mathrm{CO}_{2}$ and $\mathrm{H}_{2} \mathrm{O}$ are initially present in the domain, these species are entrained together with $\mathrm{C}_{7} \mathrm{H}_{16}$ in the HDGM regions. Diffusion of the smaller $\left(10^{-10} \mathrm{~m}\right)$ and lighter $(18 \mathrm{~kg} / \mathrm{kmol}) \mathrm{H}_{2} \mathrm{O}$ molecules from the large-density lower stream can more easily penetrate the molecular cage and interact with the other molecules than the larger $\left(6.5 \times 10^{-10} \mathrm{~m}\right)$ and heavier $(44 \mathrm{~kg} / \mathrm{kmol}) \mathrm{CO}_{2}$ molecules which have more difficulty penetrating the cage and intermixing with the other molecules. Since uphill diffusion is due to the molecular interaction among species, $\mathrm{CO}_{2}$ is more likely to experience uphill diffusion because its molecules have more time for this interaction. These effects are implicitly described at the continuum scale by the diffusion model which involves both an effective molecular collision cross-section (adapted for collisions among, rather then between, species) and the molar mass [12].

To further emphasize the difference between $\mathrm{H}_{2} \mathrm{O}$ and $\mathrm{CO}_{2}$ for the simulations in which these species are initially present, the angle between $\mathbf{J}_{\alpha}$ and $\nabla Y_{\alpha}$ is computed as a conditional average over the entire range of $|\nabla \rho|$ values for all simulations and displayed in Fig. 9 at $t_{p p}^{*}$. These results present a complementary picture to those of the PDF which were based on point-wise information, and additionally present information on the R1000p60a database. The range of $|\nabla \rho|$ values is almost twice as large for R1000p80 and R2000p60 than for R1000p60, and that for R1000p60a is almost as large as that for R2000p60 (consistent with Fig. 4b). For $\mathrm{CO}_{2}$, as seen in Fig. 9a, uphill diffusion prevails for R1000p60, R1000p80 and R2000p60, with only a minimal deviation for R1000p 80 at the largest- $|\nabla \rho|$ values, these largest- $|\nabla \rho|$ value regions being subject to the highest reaction rate. Of those simulations overwhelmingly subject to uphill diffusion, the most non-monotonic behavior encompassing a wide diversity of angles and furthest from a null angle are the R1000p80 results, in concert with the tendency for uphill diffusion to be wide-spread at high $p$. In contrast to R1000p80 and R2000p60, the results for R1000p60 show very reduced dependence of the angle on $|\nabla \rho|$, presumably because a larger $p_{0}$ or increased turbulence are conducive to larger $|\nabla \rho|$ values that are not reached in R1000p60. For all simulations in which $\mathrm{H}_{2} \mathrm{O}$ was initially present, examination of Fig. 9b shows that the angle between flux and gradient is a much stronger function of $|\nabla \rho|$ than for $\mathrm{CO}_{2}$, a fact which is attributed to the difference in molecular mass and size between these two species; since the $\mathrm{H}_{2} \mathrm{O}$ molecule has more mobility, it generates a greater diversity of angles. 
For R1000p80 and R2000p60 there is clear evidence of regular diffusion for the larger $|\nabla \rho|$ regions in which the reaction rates are larger, in accord with the above discussion. For R1000p60 the conditional average shows no regular diffusion, presumably because it does not develop the larger $|\nabla \rho|$ regions present in both R1000p80 and R2000p60; this is in concert with the information presented in Fig. 7 showing that although for the largest $|\nabla \rho|$ value the PDF indicates the occurrence of regular diffusion, the peak for uphill diffusion is very much larger, and thus on average uphill diffusion dominates.

When neither $\mathrm{H}_{2} \mathrm{O}$ nor $\mathrm{CO}_{2}$ are initially present (R1000p60a), these species only form in the flame where $T$ is large and $\rho$ is much smaller than that of the cold lower stream; then caging does not occur and thus cannot intervene to influence inter-diffusion by segregating $\mathrm{H}_{2} \mathrm{O}$ and $\mathrm{CO}_{2}$ according to molecular size and $m_{\alpha}$. Inter-species diffusion results in $\mathrm{H}_{2} \mathrm{O}$ and $\mathrm{CO}_{2}$ both diffusing, on average, through regular diffusion with the same angle of $\pi$ between $\mathbf{J}_{\alpha}$ and $\nabla Y_{\alpha}$ over the entire range of $|\nabla \rho|$ values, meaning that the peak of the PDF around $\pi$ is much larger than that around 0 .

The physical picture emerging from this analysis is that the straightforward simplicity of the results in Fig. 6 is deceptive because the details which lead to the distribution of the species in the HDGM regions are in fact quite complex. Since fuel and oxidizer are initially segregated, it is the mixing in the HDGM regions which induces the conditions conducive to reaction. Inter-species diffusion may lead to uphill diffusion which may induce liquid-phase formation [42]; caging may affect a species' ability to diffuse and experience uphill diffusion.

\subsection{Dissipation}

The dissipation, i.e. the irreversible entropy production, $g$, is a pivotal quantity in turbulence modeling: reproducing the small-scale dissipation is one of the goals of SGS modeling. According to [2],

$$
\begin{aligned}
g & =g_{\text {visc }}+g_{\text {temp }}+g_{\text {mass }}+g_{\text {reac }} \\
g_{\text {visc }} & =\frac{\mu^{v i s}}{T}\left(2 S_{i j} S_{i j}-\frac{2}{3} S_{k k} S_{l l}\right) \\
g_{\text {temp }} & =\lambda \frac{1}{T^{2}} \frac{\partial T}{\partial x_{j}} \frac{\partial T}{\partial x_{j}} \\
g_{\text {mass }} & =\frac{1}{2} \sum_{\beta=1}^{N} \sum_{\alpha=1}^{N} \frac{R_{u}}{\left(-\mathbb{D}_{\alpha \beta}\right)} \frac{\rho}{m_{\alpha} Y_{\beta}} \Pi_{\alpha \beta j} \Pi_{\alpha \beta j} \\
g_{\text {reac }} & =\frac{1}{T} \sum_{\alpha=1}^{N} \frac{\mu_{\alpha}}{m_{\alpha}} \dot{\omega}_{\alpha}
\end{aligned}
$$


where $\left(\left(-\mathbb{D}_{\alpha \beta} m\right) /\left(Y_{\alpha} m_{\beta}\right)\right)$ is a symmetric positive semi-definite matrix $[8,10]$ and

$$
\begin{aligned}
\Pi_{\alpha \beta j} & =-X_{\beta} \mathbb{D}_{\alpha \beta}\left[\left(\frac{m_{\alpha}}{m} \bar{\alpha}_{T, \beta}^{b}-\frac{m_{\beta}}{m} \bar{\alpha}_{T, \alpha}^{b}\right) \frac{1}{T} \frac{\partial T}{\partial x_{j}}+\frac{m_{\alpha} m_{\beta}}{m R_{u} T}\left(\frac{v_{\alpha}}{m_{\alpha}}-\frac{v_{\beta}}{m_{\beta}}\right) \frac{\partial p}{\partial x_{j}}\right] \\
& -\sum_{\gamma=1}^{N-1}\left(\frac{m_{\beta}}{m_{\gamma}} \mathbb{D}_{\beta \alpha} \alpha_{D \alpha \gamma}-\frac{m_{\alpha}}{m_{\gamma}} \mathbb{D}_{\alpha \beta} \alpha_{D \beta \gamma}\right)\left[\frac{\partial Y_{\gamma}}{\partial x_{j}}-Y_{\gamma} \sum_{\delta=1}^{N-1}\left(\frac{m}{m_{\delta}}-\frac{m}{m_{N}}\right) \frac{\partial Y_{\delta}}{\partial x_{j}}\right]
\end{aligned}
$$

with $\Pi_{\alpha \alpha j}=0, \Pi_{\alpha \beta j}=-\Pi_{\beta \alpha j}$.

In Fig. 10 the PDF of $g$ is shown at $t_{p p}^{*}$ for the four simulations shown in Table 1 and it is compared to the log normal distribution computed with its moments extracted from the exact PDF: that is, the shown log normal is the most accurate representation obtainable using this assumed PDF. In each case, the abscissa is $g / \max _{V}(g)$, with the understanding that $\max _{V}(g)$ has different values for each of the four simulations. The results show that in all cases the high value range of the $g / \max _{V}(g)$ is well captured by the log normal model, whereas the small-range values are less well modeled, with an indication that the representation may improve at larger $\mathrm{Re}_{0}$. Translating this information to the physical space, the regions of large dissipation are those of intense transport and/or chemical activity [1] including the diffusion flame regions, whereas the regions of small dissipation are those of smaller fluxes and/or chemical activity. The regions of smaller fluxes could be thought to be of less interest except if trace species undergo there uphill diffusion (indeed, uphill diffusion does occur in regions of small gradients [1,38]) and thus create small turbulent scales; uphill diffusion may also lead to liquid-phase formation [42]. Therefore, the trends indicate that the log normal PDF could be a useful model to represent $g$ if a method to accurately find its two moments could be developed.

\section{Summary and conclusions}

To elucidate the physics of turbulent combustion under high-pressure (high- $p$ ) conditions, a DNS database has been created using the model of high- $p$ mixing of [1] coupled with a single-step reaction involving five species: $\mathrm{C}_{7} \mathrm{H}_{16}, \mathrm{O}_{2}, \mathrm{~N}_{2}$, $\mathrm{H}_{2} \mathrm{O}$ and $\mathrm{CO}_{2}$. The configuration is that of a temporal mixing layer in which the lower and upper streams have different initial compositions. The simulations are initiated with mean profiles upon which perturbations are imposed [1] to hasten the transition to a state exhibiting turbulent characteristics, a time station labeled $t_{t r}^{*}$. Ignition is initiated at $t_{t r}^{*}$ and each simulation is pursued past the time when it displays a peak in the average-volumetric pressure,

a time station denoted by $t_{p p}^{*}$. The database encompasses the effect of $\mathrm{Re}_{0}$, of 
$p_{0}$ and of the initial composition of each of the two streams. The results were analyzed to understand important features of high- $p$ turbulent combustion.

When reaction occurs, thermodynamic energy is added to the flow. This energy is added at the smallest scales where reaction takes place and is partially backscattered; backscattered energy boosts the vortical features of the flow, as manifested by the increasingly higher rate of growth of the layer momentum thickness after the reaction is initiated. Because the peak enstrophy is reached before the peak spanwise vorticity, it is conjectured that the morphological changes in the flow through stretching and twisting are responsible for small-scale formation rather than vice versa. A simulation in which $\mathrm{H}_{2} \mathrm{O}$ and $\mathrm{CO}_{2}$ were not initially present at otherwise same initial conditions displays lagging development of small scales and morphological complexity during mixing compared to simulations in which both $\mathrm{H}_{2} \mathrm{O}$ and $\mathrm{CO}_{2}$ were initially present in the mixture so as portray Exhaust Gas Recirculation (EGR); this lag is due to lack of uphill diffusion. Once combustion is initiated, due to a larger amount of reactants leading to increased heat release which becomes transformed into dynamic energy, these turbulent characteristics catch up with those of simulations initiated with EGR.

Examination of visualizations at $t_{p p}^{*}$ shows that the main flame, corresponding to the regions of the higher temperature, occurs in the oxidizer stream, is of diffusion type and is surrounded by regions of premixed flames having lower temperature, while intense mixing continues in the fuel stream where the smaller molar mass oxidizer species diffuses into the fuel which has a larger molar mass. Combustion products are formed preponderantly in the diffusion flame. Since the flame is of partially-premixed type, the reaction rate magnitude was examined at $t_{p p}^{*}$. The results conclusively show that the higher reaction rate is achieved in the diffusion flame rather than in the premixed flame. The diffusion flame is associated with mixing and thus with density gradients; a further assessment showed that the density gradient is an increasing function of the reaction rate for all simulations. That is, the higher reaction rates occurred in the larger density-gradient magnitude regions. Simulations obtained for higher $p$ or with an initial composition where $\mathrm{H}_{2} \mathrm{O}$ and $\mathrm{CO}_{2}$ were not present showed higher reaction rates and higher density gradient magnitudes than simulations initiated with EGR at otherwise same initial conditions, or than simulations with a larger $R_{0}$ value by a factor of two at otherwise same initial conditions. The range of reaction rates in the regions of density gradients seemed independent of $\mathrm{Re}_{0}$, but what did depend on the initial Reynolds number was the magnitude of the density gradient at fixed reaction rate and this magnitude increased at larger $\mathrm{Re}_{0}$. For the present conditions, the range of the reaction rates was approximately similar for the simulation conducted at the higher pressure and for the simulation devoid of EGR effects, however at otherwise same initial conditions the density gradient magnitude was larger for the higher- $p$ simulation consistent with the reduced 
effective diffusivity at larger pressures [1].

The indication that most of the reactive activity is in HDGM regions prompted an inquiry into the evolution of their composition. Whether at a time much earlier than $t_{t r}^{*}$ or at $t_{p p}^{*}$, the dominant mass fraction in the HDGM regions is that of the fuel. Between those time stations, regions of much larger HDGM values are generally created, and the mass fraction of fuel in them increases and becomes more uniform while the mass fraction of oxygen decreases and also becomes more uniform. Since the reaction rate is larger in the regions of larger HDGM values, it is conjectured that the regions of smaller HDGM values act as a gateway from which diffusion processes bring the necessary reactants to the flame. Both $\mathrm{CO}_{2}$ and $\mathrm{H}_{2} \mathrm{O}$ form in the flame resulting in relatively small mass fractions of these species compared to the reactants. But the importance of these species is incommensurate with the small mass fractions produced. When ERG is present, $\mathrm{H}_{2} \mathrm{O}$ and $\mathrm{CO}_{2}$ experience both uphill diffusion and regular diffusion in the HDGM regions. At early times, uphill diffusion dominates for both $\mathrm{H}_{2} \mathrm{O}$ and $\mathrm{CO}_{2}$, independent of the density-gradient magnitude, this being due to the mixing of these species with the cold and dense fuel. For $\mathrm{H}_{2} \mathrm{O}$, at $t_{p p}^{*}$, in the regions of largest HDGM value, the propensity of regular diffusion is sometimes larger than that of uphill diffusion whereas this does not occur for $\mathrm{CO}_{2}$. This different behavior of these species was attributed to the disparate size of their molecules and molar masses which influence the ability of the molecule to diffuse among other molecules in a dense gas. In fact, in these simulations $\mathrm{CO}_{2}$ overwhelmingly experiences uphill diffusion, except for the simulation at the higher pressure where regular diffusion occurs only in a narrow range of regions at the larger HDGM values. In contrast, $\mathrm{H}_{2} \mathrm{O}$ experiences uphill diffusion in the smaller HDGM value regions and regular diffusion in a wider range of larger HDGM value regions than $\mathrm{CO}_{2}$; however, when regions of large HDGM value fail to become established, only uphill diffusion occurs on average. Without EGR, regular diffusion prevails for both $\mathrm{H}_{2} \mathrm{O}$ and $\mathrm{CO}_{2}$ in the entire range of HDGM values. This result is explained by the fact that without EGR both $\mathrm{H}_{2} \mathrm{O}$ and $\mathrm{CO}_{2}$ are formed and exist preponderantly in the flame which is a high-temperature region where the density is relatively low and inter-species diffusion is not restricted by the molecular size and molar mass.

Finally, the dissipation PDF follows the log normal behavior at large dissipation values, with suggestion that with increasing turbulence it may also be a good model at low dissipation. 


\section{Acknowledgments}

This work was conducted at the Jet Propulsion Laboratory (JPL) of the California Institute of Technology (Caltech) and sponsored by the Department of Energy (DOE), Basic Energy Sciences (BES) under the direction of Drs. Wade Sisk and Mark Pendersen. The contributions of Dr. Giulio Borghesi and Dr. Kenneth G. Harstad are acknowledged. The computational resources were provided by the NASA Advanced Supercomputing at Ames Research Center under the Aeronautics Research Mission Directorate program (Drs. Jeff Moder and Michael Rogers) and by National Energy Research Supercomputing Center of the Department of Energy.

\section{A Transport properties}

Unlike for atmospheric- $p$ flows where three transport properties are generally sufficient (viscosity, diffusivity and thermal conductivity), for high- $p$ conditions there are four relevant transport properties.

\section{A.1 Mixture viscosity}

To compute the individual species viscosity, $\mu_{\alpha}$, the Lucas method [49] has been selected due to its high- $p$-accuracy capabilities. To compute the mixture physical viscosity, $\mu_{p h}$, the Wilke method [49] is utilized providing

$$
\begin{aligned}
\mu_{p h} & =\sum_{\alpha=1}^{N} X_{\alpha} \omega_{\alpha}^{M} \mu_{\alpha} \\
\left(\omega_{\alpha}^{M}\right)^{-1} & =\sum_{\beta=1}^{N} \phi_{\alpha \beta} X_{\beta} \\
\phi_{\alpha \beta} & =\frac{\left[1+\left(\mu_{\alpha} / \mu_{\beta}\right)^{1 / 2}\left(m_{\beta} / m_{\alpha}\right)^{1 / 4}\right]^{2}}{\left[8\left(1+m_{\alpha} / m_{\beta}\right)\right]^{1 / 2}}
\end{aligned}
$$

where $\omega_{\alpha}^{M}$ are weighting factors [49]. Different from $\mu_{p h}$ is the reference viscosity $\mu_{R}$ defined in section 4 and the computational viscosity $\mu$ used to enable resolution to scales of $O\left(\eta_{K}\right)$, as explained in section 4 . 


\section{A.2 Mixture thermal conductivity}

To compute the physical mixture thermal conductivity, $\lambda_{p h}$, first the species conductivities $\lambda_{\alpha}$ are calculated using the Stiel-Thodos method [49], and then the Wassiljewa-Mason-Saxena method [49] is utilized to compute $\lambda_{p h}$ from $\lambda_{\alpha}$ as

$$
\lambda_{p h}=\sum_{\gamma=1}^{N} X_{\alpha} \omega_{\alpha}^{Q} \lambda_{\alpha}
$$

where

$$
\omega_{\alpha}^{Q}=\omega_{\alpha}^{M}
$$

The computation of a scaled thermal conductivity, $\lambda$, used to perform DNS is explained in section 4 .

\section{A.3 Binary mass diffusivities}

Matrix elements $\mathcal{D}_{\alpha \gamma}^{b}$ are the building blocks of $\mathbb{D}_{\alpha \gamma}$ and ultimately of $D_{\alpha \gamma}^{\prime}$. To compute $\mathcal{D}_{\alpha \gamma}^{b}=\mathcal{D}_{\alpha \gamma}$, the method of [12] is adopted that gives (in cgs units)

$$
n \mathcal{D}_{\alpha \gamma}=2.81 \times 10^{-5} \frac{f_{D, \alpha \gamma}(T)}{r_{D} v_{c, \alpha \gamma}^{2 / 3}}\left[\left(\frac{1}{m_{\alpha}}+\frac{1}{m_{\gamma}}\right) T\right]^{1 / 2}
$$

where $f_{D, \alpha \gamma}(T)$ is generically defined for each matrix element as $f_{D}(T) \equiv$ $\left(T_{\text {red }}\right)^{s}$ with $\ln s=\sum_{\zeta=0}^{5} a_{\zeta}^{s}\left(\ln T_{r e d}\right)^{\zeta}$ where the $a^{s}$ vector has elements $\{-$ $0.84211,-0.32643,-0.10053,0.07747,0.0127,-0.00995\}$, and $r_{D}$ is a constant $O(1)$ which provides an empirical adjustment for the specifics of the collisional interactions of a selected pair of species. $T_{r e d, \alpha \gamma} \equiv T / T_{c, \alpha \gamma}$ with $T_{c, \alpha \gamma}$ defined in Appendix B; $v_{c, \alpha \gamma}$ is defined in Appendix B as well. Values of $r_{D}$ are listed elsewhere [12] for species pairs relevant to combustion.

\section{A.4 Binary thermal diffusion factors}

According to [11]

$$
\begin{aligned}
\alpha_{T, \alpha \gamma}^{b} & =\zeta_{\alpha \gamma} \frac{\left(m_{\alpha} \omega_{\gamma}^{T}-m_{\gamma} \omega_{\alpha}^{T}\right)}{\left(m_{\alpha}+m_{\gamma}\right) \mathcal{D}_{\alpha \gamma}} \\
\omega_{\alpha}^{T} & =\frac{\omega_{\alpha}^{Q} \lambda_{\alpha}}{R_{u} n}, \quad \zeta_{\alpha \gamma}=\frac{6}{5} C_{\alpha \gamma}^{*}-1
\end{aligned}
$$

where $\omega_{\alpha}^{Q}$ is computed from equations (A.2) and (A.5), and $C_{\alpha \gamma}^{*}$ is given by [18] and is function of a normalized temperature including the characteristic molecular interaction potential [11]. 


\section{B Equation of state relationships}

Miscellaneous relationships relevant to the EOS are

$$
a_{m i x}=\sum_{\alpha} \sum_{\gamma} X_{\alpha} X_{\gamma} a_{\alpha \gamma}(T), \quad b_{m i x}=\sum_{\alpha} X_{\alpha} b_{\alpha}
$$

where indices do not follow the Einstein notation, and

$$
\begin{aligned}
a_{\alpha \gamma} & =\left(1-k_{\alpha \gamma}^{\prime}\right) \sqrt{\alpha_{\alpha \alpha} \alpha_{\gamma \gamma}}, \\
\alpha_{\alpha \alpha}(T) & \equiv 0.457236\left(R_{u} T_{c, \alpha}\right)^{2} \times \frac{\left[1+c_{\alpha}\left(1-\sqrt{T_{r e d, \alpha}}\right)\right]^{2}}{p_{c, \alpha}} \\
c_{\alpha} & =0.37464+1.54226 \Omega_{\alpha}-0.26992 \Omega_{\alpha}^{2}
\end{aligned}
$$

where $T_{r e d, \alpha} \equiv T / T_{c, \alpha}, T_{c, \alpha}$ is the critical temperature and $\Omega_{\alpha}$ is the acentric factor. Also,

$$
\begin{gathered}
b_{\alpha}=0.077796 \frac{R_{u} T_{c, \alpha}}{p_{c, \alpha}} \\
T_{c, \alpha \gamma}=\left(1-k_{\alpha \gamma}\right) \sqrt{T_{c, \alpha} T_{c, \gamma}} \text { with } k_{\alpha \alpha}=0 \\
v_{c, \alpha \gamma}=\frac{1}{8}\left(v_{c, \alpha}^{1 / 3}+v_{c, \gamma}^{1 / 3}\right)^{3}, \quad Z_{c, \alpha \gamma}=\frac{1}{2}\left(Z_{c, \alpha}+Z_{c, \gamma}\right), \quad p_{c, \alpha \gamma}=\frac{R_{u} T_{c, \alpha \gamma} Z_{c, \alpha \gamma}}{v_{c, \alpha \gamma n}}
\end{gathered}
$$

with $T_{r e d, \alpha \gamma} \equiv T / T_{c, \alpha \gamma}, Z_{c, \alpha}$ being the critical compression factor with the compression factor defined as $Z=p /\left(\rho T R_{u} / m\right), v_{c, \alpha}$ being the critical volume, and $p_{c, \alpha}$ being the critical pressure. $k_{\alpha \gamma}$ is an empirical mixing parameter. The relationship between parameters $k_{\alpha \gamma}$ and $k_{\alpha \gamma}^{\prime}$ is

$$
\left(1-k_{\alpha \gamma}\right)=\left(1-k_{\alpha \gamma}^{\prime}\right) \frac{\left(v_{c, \alpha} v_{c, \gamma}\right)^{1 / 2}}{v_{c, \alpha \gamma}} \frac{Z_{c, \alpha \gamma}}{\sqrt{Z_{c, \alpha} Z_{c, \gamma}}} .
$$

Values of $k_{\alpha \gamma}^{\prime}$ are listed in Table 4, where the values were obtained from [49] or [50]. For all pairs not in Table $4, k_{\alpha \gamma}^{\prime}=0$ is used.

\section{References}

[1] E. Masi, J. Bellan, K. Harstad, N. Okong'o, Multi-species turbulent mixing under supercritical-pressure conditions: modeling, Direct Numerical Simulation and analysis revealing species spinodal decomposition, J. Fluid Mech. 721 (2013) 578-626. 
[2] G. Borghesi, J. Bellan, Irreversible entropy production rate in high-pressure turbulent reactive flows,. Proc. Comb. Inst. 35 (2015) 1537-1547.

[3] T. Edwards, Cracking and deposition behavior of supercritical hydrocarbon aviation fuels, Combust. Sci. Technol. 178 (2006)307-334.

[4] R. Keppeler, E. Tangermann, U. Allaudin, M. Pfitzner, Large Eddy Simulation of low to high turbulent combustion in an elevated pressure environment, Flow Turbulence Combust. 92 (2014) 767-802.

[5] G. Lacaze, J. C. Oefelein, A non-premixed combustion model based on flame structure analysis at supercritical pressures. Combustion and Flame, 159 (2012) 2087-2103.

[6] X. Petit, G. Ribert, Lartigue, G., P. Domingo, Large Eddy Simulation of supercritical fluid injection, J. Supercritical Fluids, 84 (2013) 61-73.

[7] H. Müller, C. A. Niedermeier, J. Matheis, M. Pfitzner, S. Hickel, Large-eddy simulation of nitrogen injection at trans- and supercritical conditions, Phys. Fluids, 28 (2016) 015102.

[8] V. Giovangigli, L. Matuszewsky, F. Dupoirieux, Detailed modeling of planar transcritical H2-O2-N2 flames, Comb. Th. Mod. 15 (2011) 141-182.

[9] P. Moin, K. Mahesh, Direct Numerical Simulation: A Tool in Turbulence Research, Annual Review of Fluid Mechanics, 30 (1998) 539-578.

[10] J. Keizer, Statistical Thermodynamics of Nonequilibrium Processes,SpringlerVerlag, New York, 1987.

[11] K. Harstad, J. Bellan, Mixing rules for multicomponent mixture mass diffusion coefficients and thermal diffusion factors, J. Chem. Phys. 120(12) (2004) 56645673 .

[12] K. Harstad, J. Bellan, High-pressure binary mass-diffusion coefficients for combustion applications, Ind. \& Eng. Chem. Res. 43(2) (2004) 645-654.

[13] D.J. Pine, N. Easwar, J.V. Maher, W.I. Goldburg, Turbulent suppression of spinodal decomposition, Phys. Rev. A. 29(1), (1984) 308-313.

[14] L.C. Selle, N.A. Okong'o, J. Bellan, K.G. Harstad, Modeling of subgrid scale phenomena in supercritical transitional mixing layers: an a priori study, J. Fluid Mech. 593 (2007) 57-91.

[15] F.N. Egolfopoulos, P.E. Dimotakis, Non-Premixed hydrocarbon ignition at high strain rates, Symp. (Int.) Combust. 27 (1998) 641-648.

[16] C.G. Fotache, T.G. Kreutz, C.K. Law, Ignition of counterflowing methane versus heated air under reduced and elevated pressures, Combust. Flame 108 (1997) 442-470.

[17] N. Okong'o, J. Bellan, Direct numerical simulation of a transitional supercritical binary mixing layer: heptane and nitrogen, J. Fluid Mech. 464 (2002) 1-34. 
[18] J. Hirshfelder, C. Curtis, R. Bird, Molecular Theory of Gases and Liquids, John Wiley and Sons, 1964.

[19] A. Ern, V. Giovangigli, Multicomponent transport algorithms, Lecture Notes in Physics m24, Springer Verlag, 1994.

[20] R.J. Kee, M.E. Coltrin, P. Glarborg, Chemically reactive flow. Theory and Practice, John Wiley and Sons, 2005

[21] A. Ern, V. Giovangigli, Thermal diffusion effects in hydrogen-air and methaneair flames, Combust. Theory Modelling 2 (1998) 349-372.

[22] N. Okong'o, K. Harstad, J. Bellan, Direct numerical simulations of $\mathrm{O}_{2} / \mathrm{H}_{2}$ temporal mixing layers under supercritical conditions, AIAA J. 40(5) (2002) 914-926.

[23] M R Overholt, S B. Pope, Direct numerical simulation of a statistically stationary, turbulent reacting flow, Combust. Theory Modelling 3 (1999) 371408.

[24] M.Mortensen , S.M. de Bruyn Kops, C.M. Cha, Direct numerical simulations of the double scalar mixing layer Part II: Reactive scalars, Comb. Flame 149 (2007) 392-408.

[25] K. Harstad, R. S. Miller, J. Bellan, Efficient high pressure state equations, AIChE J. 43 (1997) 1605-1610.

[26] C. Westbrook, F.L. Dryer, Simplified reaction mechanisms for the oxidation of hydrocarbon fuels in flames, Combust. Sci. Techn. 27 (1981) 31-43.

[27] E. Mastorakos, Ignition of turbulent non-premixed flames, Prog. Ener. Combust. Sci. 35 (2009) 57-97.

[28] E. Mastorakos, T.A. Baritaud, T.J. Poinsot, Numerical simulations of autoignition in turbulent mixing flows, Combust. Flame 109 (1997) 198-223.

[29] S. Sreedhara, K.N. Lakshmisha, 2000 Direct numerical simulation of autoignition in a non-premixed, turbulent medium, Proc. Combust. Inst. 28(1) (2000) 25-33.

[30] G. Borghesi, E. Mastorakos, R.S. Cant, Complex chemistry DNS of n-heptane spray autoignition at high pressure and intermediate temperature conditions, Combust. Flame 160 (2013) 1254-1275.

[31] N. Okong'o, J. Bellan, Consistent boundary conditions for multicomponent real gas mixtures based on characteristic waves, J. Comp. Phys. 176 (2002) 330-344.

[32] G.K. Batchelor, An Introduction to Fluid Dynamics, Cambridge University Press, 1999.

[33] R. Moser, M. Rogers, Mixing transition and the cascade to small scales in a plane mixing layer, Phys. Fluids A3(5) (1991) 1128-1134. 
[34] C. Kennedy, M. Carpenter, Several new numerical methods for compressible shear layer simulations, Appl. Num. Math. 14 (1994) 397-433.

[35] S.M. Muller, D. Scheerer, A method to parallelize tridiagonal solvers, Parallel Computing17 (1991) 181-188.

[36] G. Borghesi, J. Bellan, A priori and a posteriori investigations for developing Large Eddy Simulations of multi-species turbulent mixing under high-pressure conditions, Phys. Fluids 27 (2015) 035117 (35 pages).

[37] A.I Olemskol, E.A. Toropov, I.A. Sklyar, Self-consistent theory of the transition of an unstable thermodynamic system from spinodal to heterophase kinetics, Sov. Phys. JETP 73(3) (1991) 545-551.

[38] R.W. Balluffi, S.M. Allen, W.C. Carter, Kinetics of Materials, John Wiley \& Sons, Inc., 2005.

[39] M. Oschwald, A. Schik, Supercritical nitrogen free jet investigated by spontaneous Raman scattering, Exp. Fluids. 27 (1999) 497-506.

[40] B. Chehroudi, D. Talley, E. Coy, Visual characteristics and initial growth rates of round cryogenic jets at subcritical and supercritical pressures, Phys. Fluids. 14(2) (2002) 850-861.

[41] I.A. Hannoun, H.J.S. Fernando, E. J. List, Turbulence structure near a sharp density interface, J. Fluid Mech. 189 (1998) 189-209.

[42] E.B Nauman, D. Q. He, Nonlinear diffusion and phase separation, Chem. Eng. Sci. 56 (2001) 1999-2018.

[43] Y. Zhang, Geochemical kinetics, Princeton University Press, 2008.

[44] R. Taylor, R. Krishna, Multicomponent Mass Transfer, John Wiley \& Sons, Inc., 1993.

[45] J.W. Cahn, J.E. Hilliard, Free energy of a nonuniform system. I. Interfacial free energy, J. Chem. Phys. 28(2) (1958) 258-267.

[46] I.L. Pioro, H.F. Khartabil, R.B. Duffey, Heat transfer to supercritical fluids flowing in channels - empirical correlations (survey), Nuclear Engineering and Design 230(1-3) (2004) 69-91.

[47] J.Y Yoo, The turbulent flows of supercritical fluids with heat transfer, Annu. Rev. Fluid Mech. 45 (2013) 495-525.

[48] H. Yamashita, M. Shimada, T. Takeno, A numerical study on flame stability at the transition point of jet diffusion flames, Symp. (Int.) Combust. 26 (1996) $27-34$.

[49] R.C. Reid, J.M. Prausnitz, B.E. Polling, The Properties of Gases and Liquids, 4th Edition, McGraw-Hill Book Company, 1987.

[50] H. Knapp, R. Döring, L. Oellrich, U. Plöcker, J.M. Prausnitz, R. Langhorst, S. Zeck, Vapor-Liquid Equilibria for Mixtures of Low Boiling Substances, DECHMA Chem. Data Series, 6 DECHMA, Frankfurt, 1982. 


\begin{tabular}{cccccccccccc}
\hline Run & $\operatorname{Re}_{0}$ & $\mathcal{F}$ & $p_{0}$ & $\left(\frac{\rho_{L}}{\rho_{U}}\right)_{0} N_{1} \times N_{2} \times N_{3}$ & $\begin{array}{c}\Delta x \\
(\mathrm{~atm})\end{array}$ & $t_{t r}^{*}$ & $\operatorname{Re}_{m, t r} t_{p p}^{*}$ & $\operatorname{Re}_{m, p p}$ \\
\multicolumn{7}{c}{} & \multicolumn{7}{c}{$\left(10^{-4} \mathrm{~m}\right)$} \\
\hline R1000p60 & 1000 & 7751 & 60 & 9.68 & $480 \times 530 \times 288$ & 4.17 & 90 & 1766 & 134 & 2221 \\
R1000p80 & 100011065 & 80 & 10.03 & $584 \times 630 \times 344$ & 3.42 & 85 & 1749 & 125 & 2106 \\
R1000p60a 100010331 & 60 & 12.56 & $480 \times 530 \times 288$ & 4.17 & 90 & 1762 & 136 & 2218 \\
R2000p60 & 2000 & 3875 & 60 & 9.68 & $768 \times 804 \times 460$ & 2.60 & 117 & 3804 & 171 & 4106 \\
\hline
\end{tabular}

Table 1

List of the Direct Numerical Simulation realizations and associated resolution. $L_{i}$ is the size of the domain in the $x_{i}$ direction, in meters. For all layers, $L_{1}=0.2 \mathrm{~m}$ and $L_{3}=0.12 \mathrm{~m}$. For all $R e_{0}=1000 \mathrm{DNS}, F_{3 D}=0.048, F_{2 D}=0.05$ and $L_{2}=0.221 \mathrm{~m}$ for $p=60 \mathrm{~atm}$ and $L_{2}=0.216 \mathrm{~m}$ for $p=80 \mathrm{~atm}$; for $R e_{0}=2000, F_{3 D}=0.012, F_{2 D}=0.03$ and $L_{2}=0.209 \mathrm{~m}$. For all simulations $T_{U}=1000 \mathrm{~K}, T_{L}=600 \mathrm{~K}$. Information regarding the initial upper stream and lower stream compositions is provided in table 2. The subscripts $t r$ and $p p$ denote the transitional time and pressure-peak time, respectively.

\begin{tabular}{ccccccc}
\hline Run & Stream & $Y_{\mathrm{H}_{2} \mathrm{O}}$ & $Y_{\mathrm{CO}_{2}}$ & $Y_{\mathrm{O}_{2}}$ & $Y_{C_{7} \mathrm{H}_{16}}$ & $Y_{N_{2}}$ \\
\hline All simulations & $U$ & 0.01 & 0.035 & 0.20 & 0.00 & 0.755 \\
(except R1000p60a) & $L$ & 0.01 & 0.035 & 0.00 & 0.955 & 0.00 \\
\hline R1000p60a & $U$ & 0.00 & 0.00 & 0.245 & 0.00 & 0.755 \\
& $L$ & 0.00 & 0.00 & 0.00 & 1.00 & 0.00 \\
\hline
\end{tabular}

Table 2

Initial mass fractions of water, carbon dioxide, oxygen, n-heptane and nitrogen in the upper and lower streams.

Table 3

\begin{tabular}{lllllll}
\hline Species & $\begin{array}{l}\text { Species } \\
\text { label }(\alpha)\end{array}$ & $\begin{array}{l}m_{\alpha} \\
(\mathrm{kg} / \mathrm{kmol})\end{array}$ & $\begin{array}{l}T_{c} \\
(\mathrm{~K})\end{array}$ & $\begin{array}{l}p_{c} \\
(\mathrm{bar})\end{array}$ & $\begin{array}{l}v_{c} \\
\left(\frac{10^{-3} \mathrm{~m}^{3}}{\mathrm{kmol}}\right)\end{array}$ & \\
\hline $\mathrm{H}_{2} \mathrm{O}$ & 1 & 18.015 & 647.3 & 221 & 57.1 & 0.344 \\
$\mathrm{CO}_{2}$ & 2 & 44.01 & 304.1 & 73.8 & 93.9 & 0.239 \\
$\mathrm{O}_{2}$ & 3 & 32.0 & 154.6 & 50.43 & 73.4 & 0.025 \\
$\mathrm{C}_{7} \mathrm{H}_{16}$ & 4 & 100.2 & 540.2 & 27.4 & 432 & 0.349 \\
$\mathrm{~N}_{2}$ & 5 & 28.013 & 126.26 & 33.4 & 89.8 & 0.039 \\
\hline
\end{tabular}

Species properties. The species are listed in order of index $\alpha . \Omega$ is the acentric factor. 


\begin{tabular}{r|ccccccc}
\hline$\alpha$ & alkane & alkane & alkane & alkane & $\mathrm{CO}_{2}$ & $\mathrm{CO}_{2}$ & $\mathrm{H}_{2} \mathrm{O}$ \\
$\gamma$ & alkane & $\mathrm{N}_{2}, \mathrm{O}_{2}$ & $\mathrm{CO}_{2}$ & $\mathrm{H}_{2} \mathrm{O}$ & $\mathrm{H}_{2} \mathrm{O}$ & $\mathrm{N}_{2}, \mathrm{O}_{2}$ & $\mathrm{~N}_{2}, \mathrm{O}_{2}$ \\
$k^{\prime}$ & 0.0 & 0.15 & 0.11 & $0.093-0.006 n_{c}$ & 0.095 & -0.017 & 0.17 \\
\hline
\end{tabular}

Values of $k^{\prime}$ for species pairs. $n_{c}$ is the number of $\mathrm{C}$ atoms in the species. 

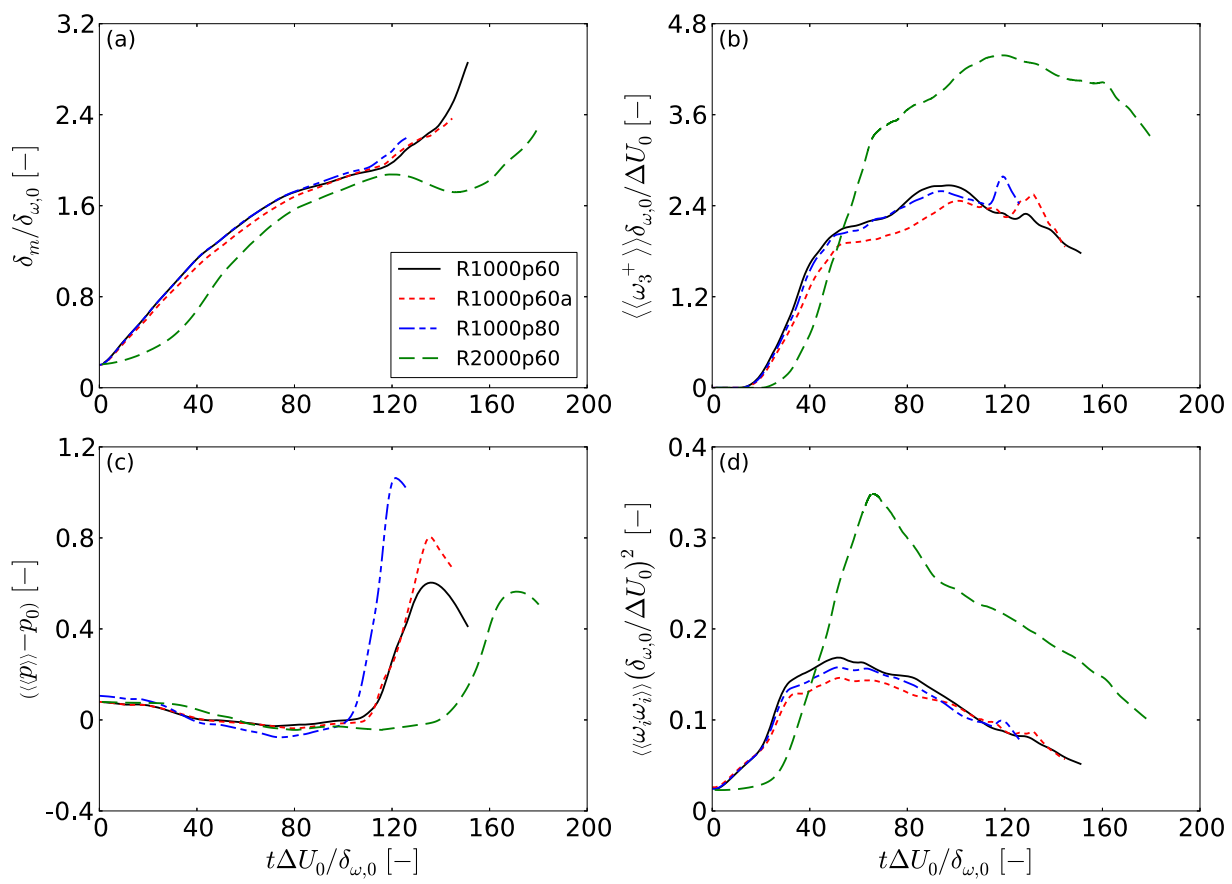

Fig. 1. Time-wise evolution of integral quantities (a) $\delta_{m} / \delta_{\omega, 0}$, (b) $\left\langle\left\langle\omega_{3}^{+}\right\rangle\right\rangle \delta_{\omega, 0} / \Delta U_{0}$, and $(\mathrm{c})\left(\langle\langle p\rangle\rangle-p_{0}\right)$ and $(\mathrm{d})\left\langle\left\langle\omega_{i} \omega_{i}\right\rangle\right\rangle\left(\delta_{\omega, 0} / \Delta U_{0}\right)^{2}$; simulations listed in Table 1. 

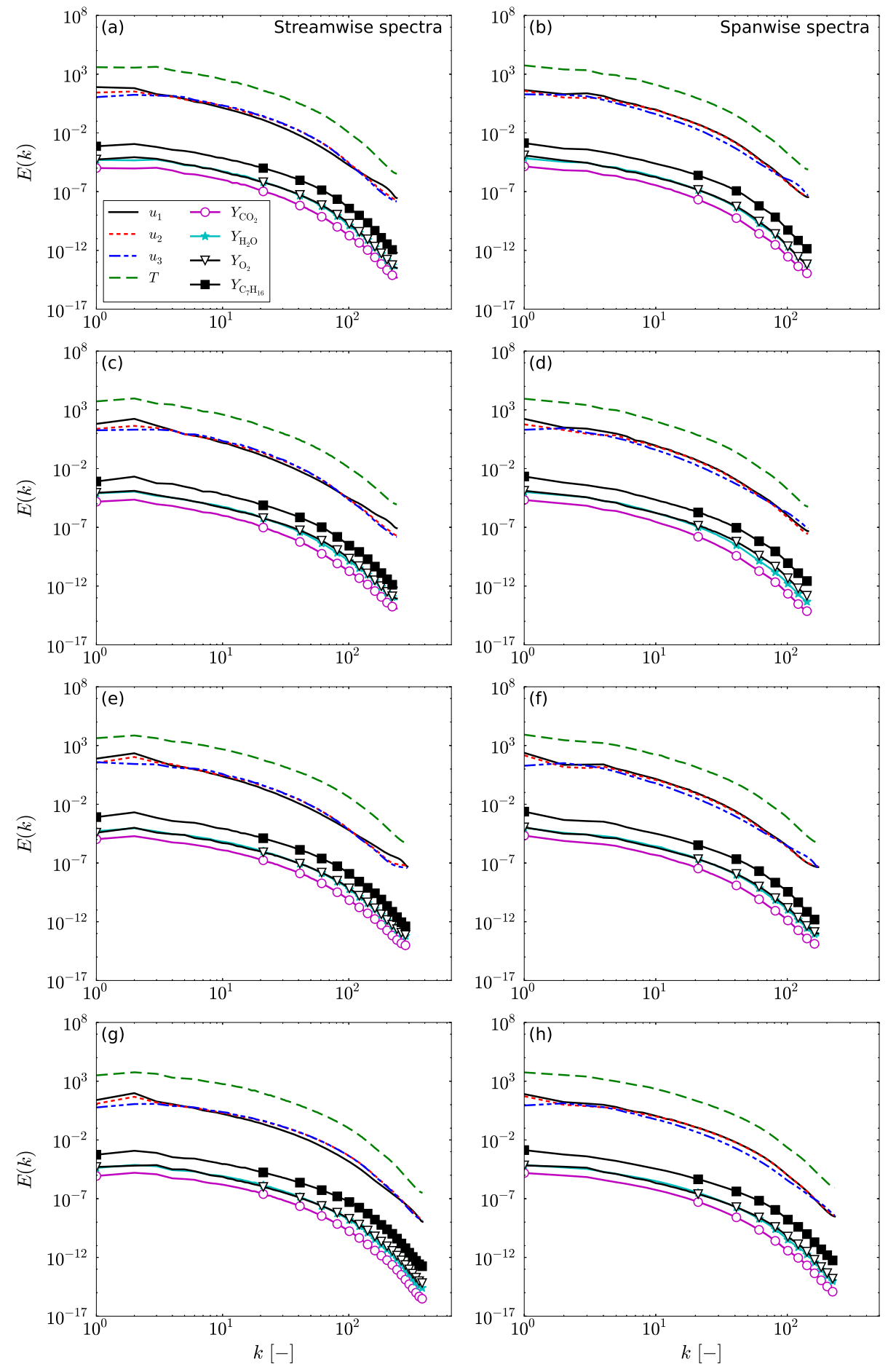

Fig. 2. One-dimensional spectra at $t_{t r}^{*}$ for several simulations: (a,b) R1000p60, (c,d) R1000p60a, (e,f) R1000p80 and (g,h) R2000p60. 

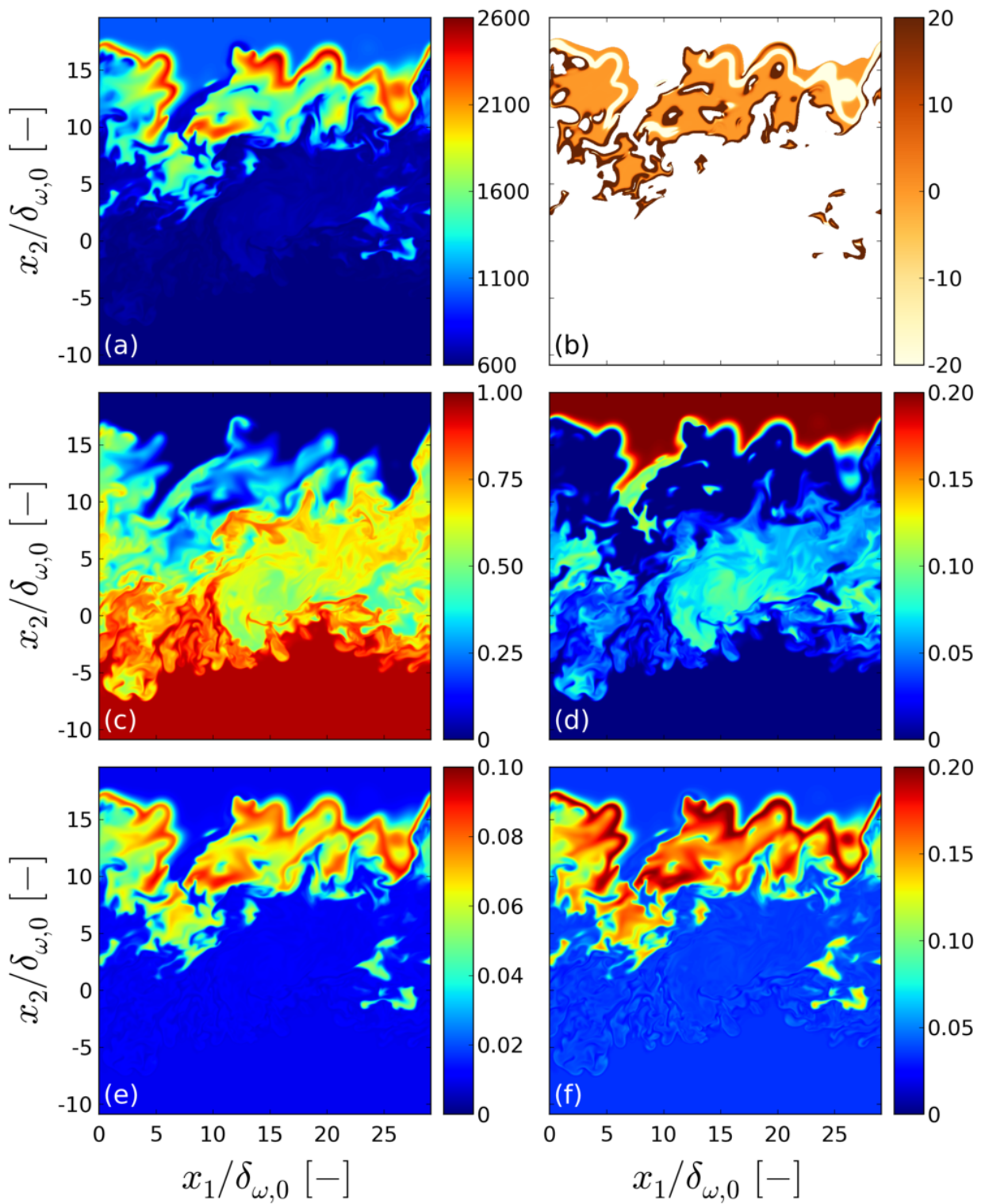

Fig. 3. Visualizations at $t_{p p}^{*}$ in the $x_{3} / L_{3}=1 / 16$ plane of (a) $T(\mathrm{~K})$, (b) the Flame Index, (c) $Y_{\mathrm{C}_{7} \mathrm{H}_{16}}$, (d) $Y_{\mathrm{O}_{2}}$, (e) $Y_{\mathrm{H}_{2} \mathrm{O}}$ and (f) $Y_{\mathrm{CO}_{2}}$. All for R2000p60. 

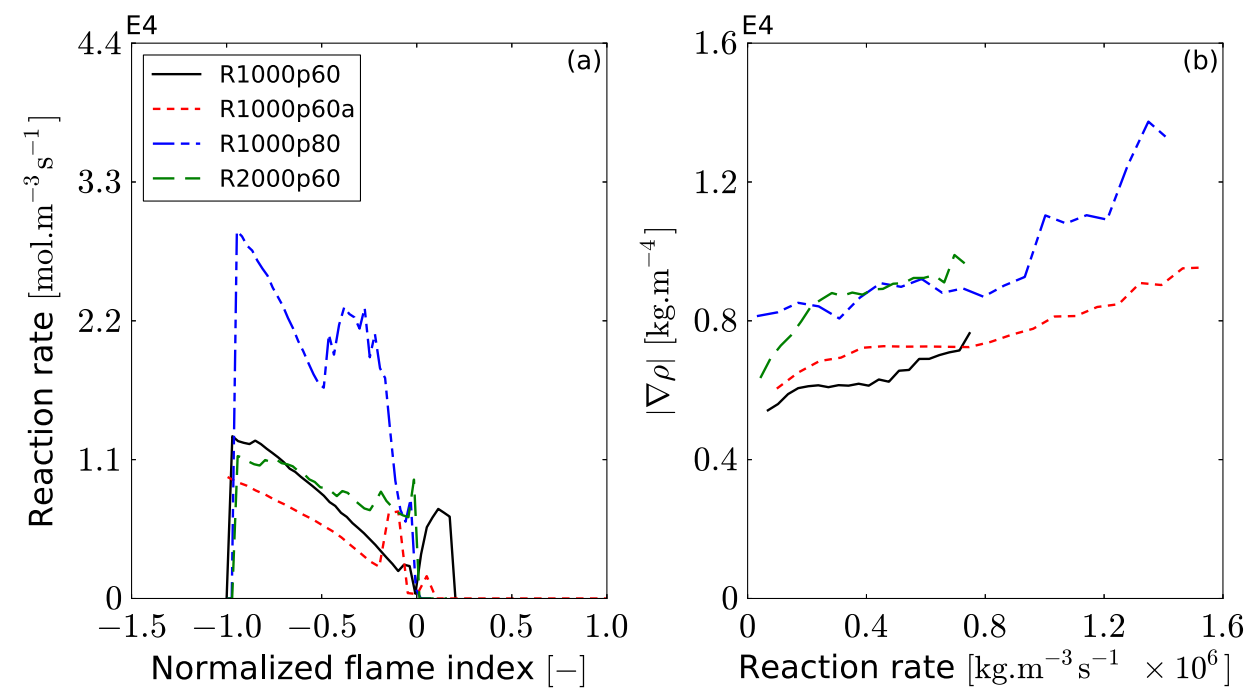

Fig. 4. Conditional averages at $t_{p p}^{*}$ of (a) $\dot{\omega}$ conditioned on a normalized Flame Index, and (b) $|\nabla \rho|$ conditioned on $\dot{\omega}$. In (a) $\dot{\omega}$ is restricted to values larger than $\dot{\omega}_{\max _{V}} \times 10^{-2}\left(\mathrm{~mol} \mathrm{~m}^{-3} \mathrm{~s}^{-1}\right)$ for each of the DNS, where $V$ is the computational domain volume.

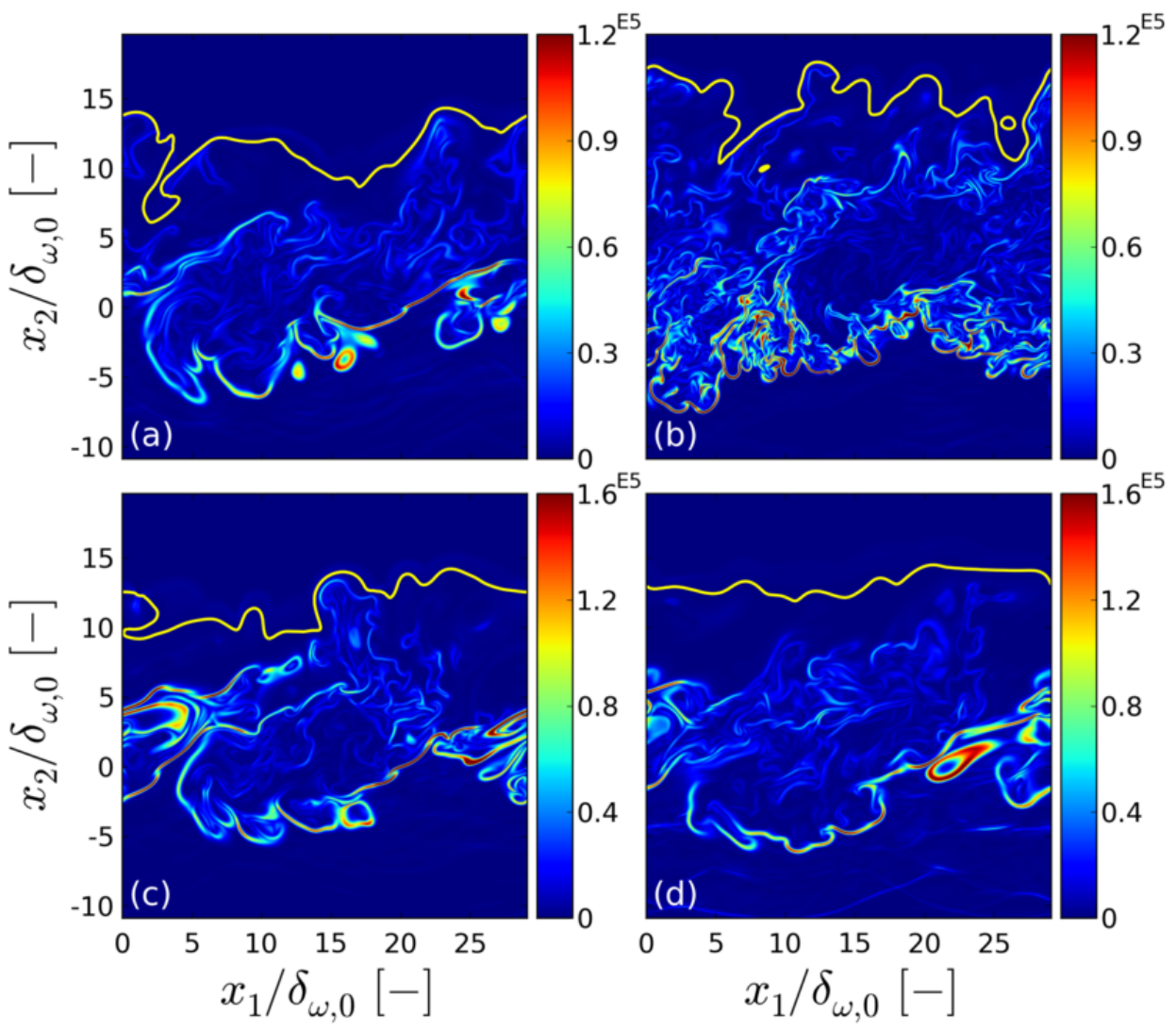

Fig. 5. $|\nabla \rho|\left(\mathrm{kg} / \mathrm{m}^{4}\right)$ at $t_{p p}^{*}$ in the $x_{3} / L_{3}=1 / 16$ plane for (a) R1000p60, (b) R2000p60, (c) R1000p80 and (d) R1000p60a. The yellow contour indicates the stoichiometric locus. 

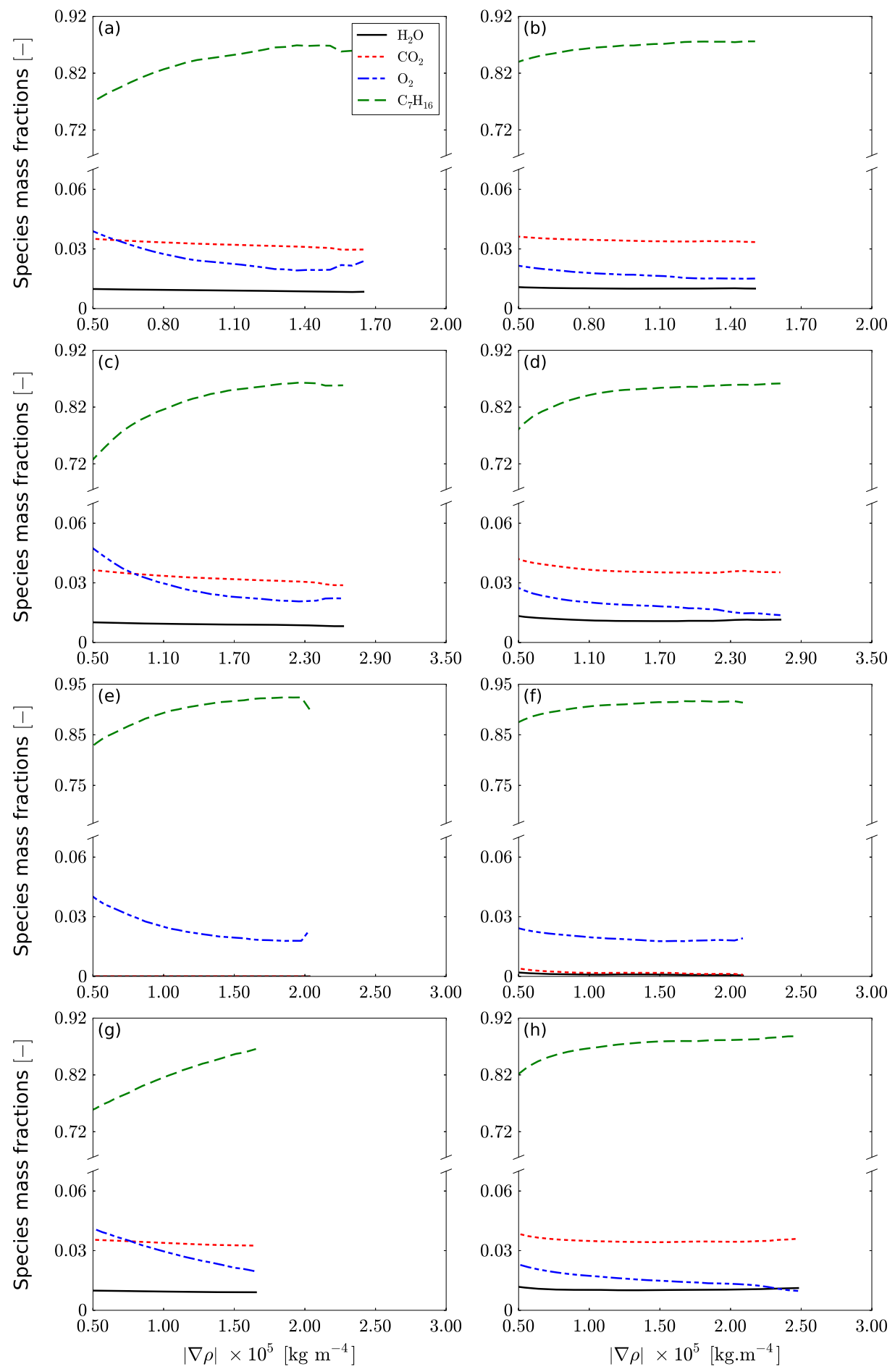

Fig. 6. Conditional averages of the composition of the HDGM regions at $t^{*}=30$ (a,c,e,g) and at the respective $t_{p p}^{*}(\mathrm{~b}, \mathrm{~d}, \mathrm{f}, \mathrm{h}) .(\mathrm{a}, \mathrm{b}) \mathrm{R} 1000 \mathrm{p} 60,(\mathrm{c}, \mathrm{d}) \mathrm{R} 1000 \mathrm{p} 80,(\mathrm{e}, \mathrm{f})$ R1000p60a and (g,h) R2000p60. 

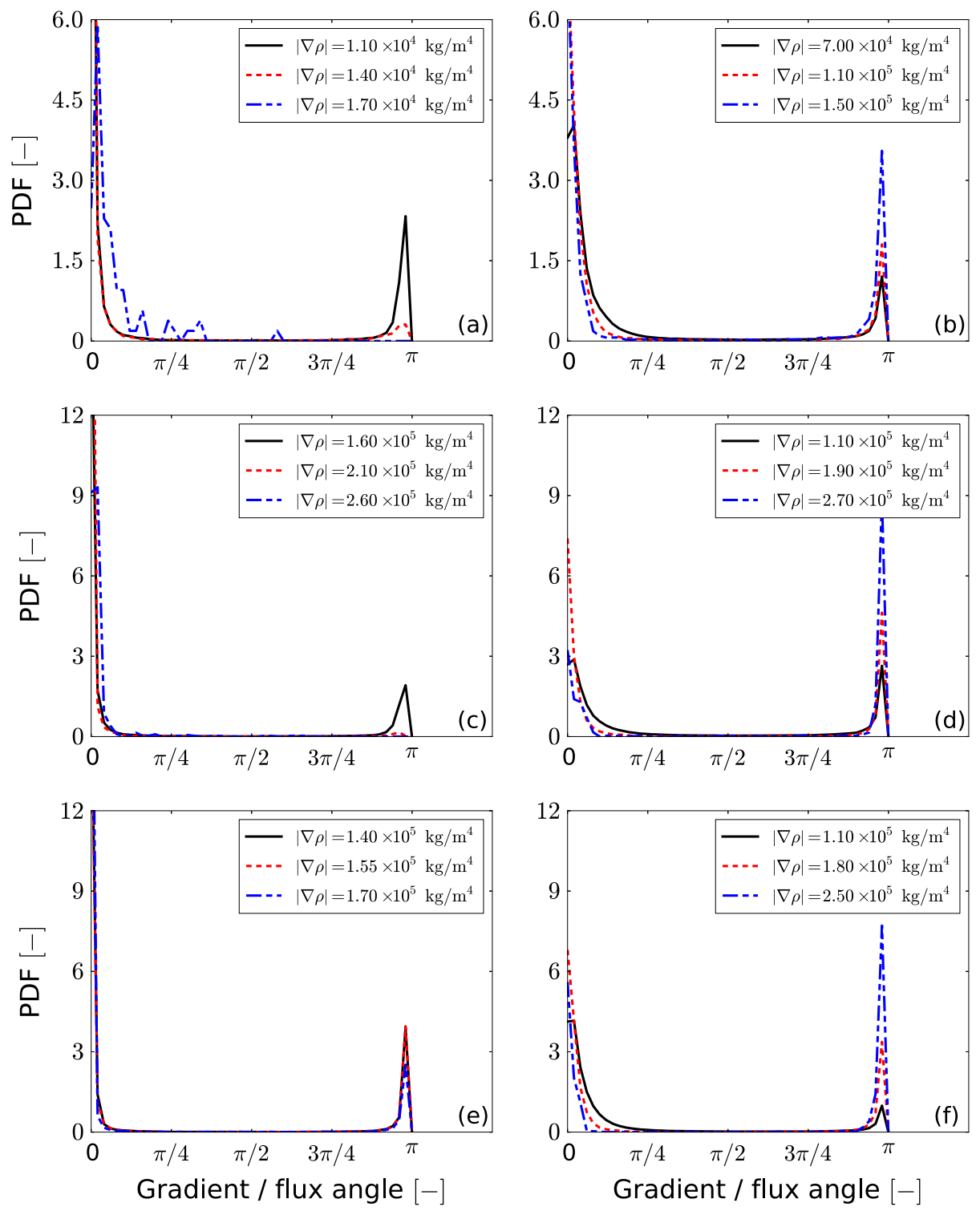

Fig. 7. Probability density function of the angle between $\nabla Y_{\mathrm{H}_{2} \mathrm{O}}$ and $\mathbf{J}_{\mathrm{H}_{2} \mathrm{O}}$ at $t^{*}=30$ $(\mathrm{a}, \mathrm{c}, \mathrm{e})$ and $t_{p p}^{*}(\mathrm{~b}, \mathrm{~d}, \mathrm{f})$ for (a,b) R1000p60, (c,d) R1000p80 and (e,f) R2000p60. 

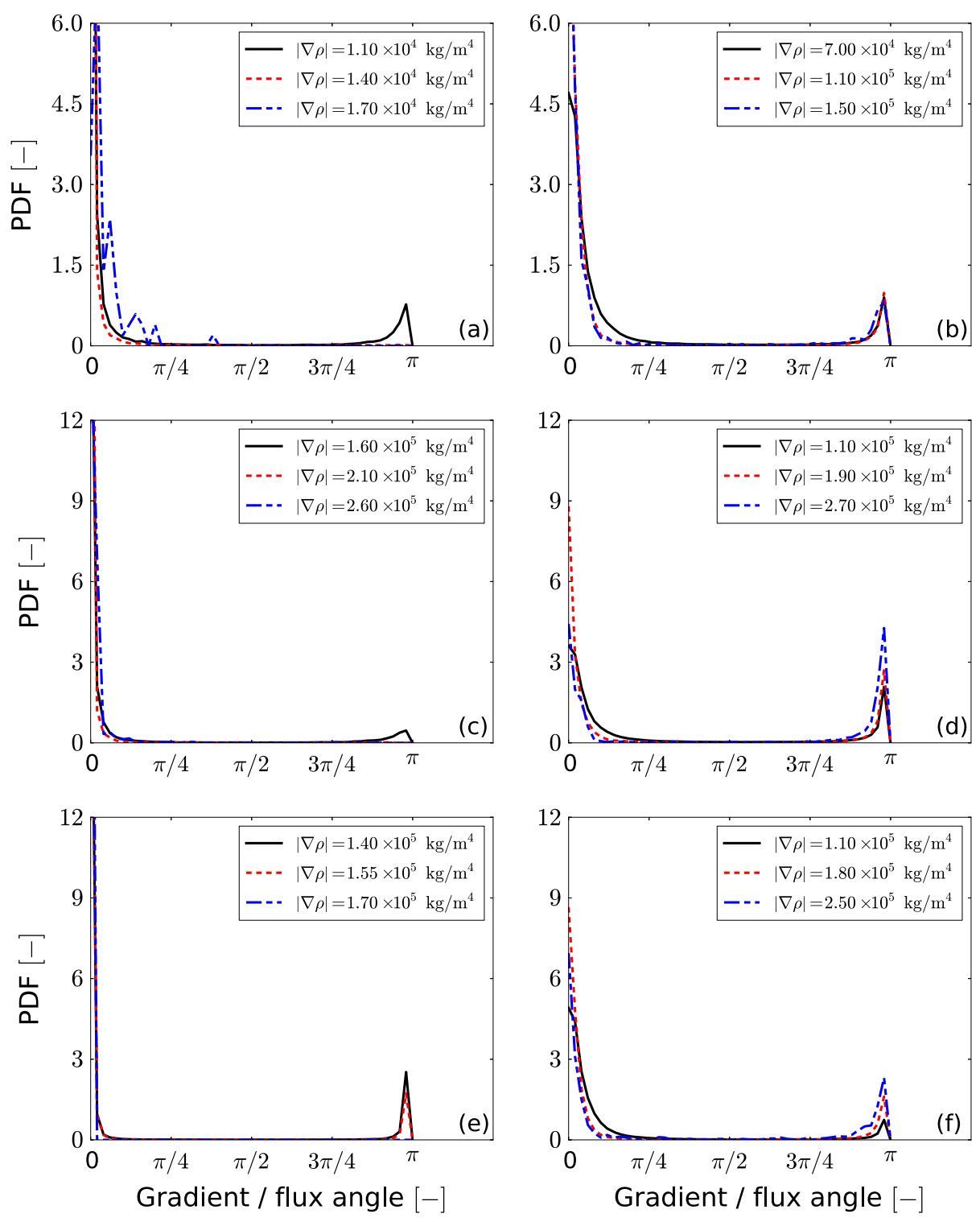

Fig. 8. Probability density function of the angle between $\nabla Y_{\mathrm{CO}_{2}}$ and $\mathbf{J}_{\mathrm{CO}_{2}}$ at $t^{*}=30$ $(\mathrm{a}, \mathrm{c}, \mathrm{e})$ and $t_{p p}^{*}(\mathrm{~b}, \mathrm{~d}, \mathrm{f})$ for (a,b) R1000p60, (c,d) R1000p80 and (e,f) R2000p60. The rms of the source terms have have been non-dimensionalized by $\left(\Delta U_{0} / \delta_{\omega, 0}\right)^{3}$. 

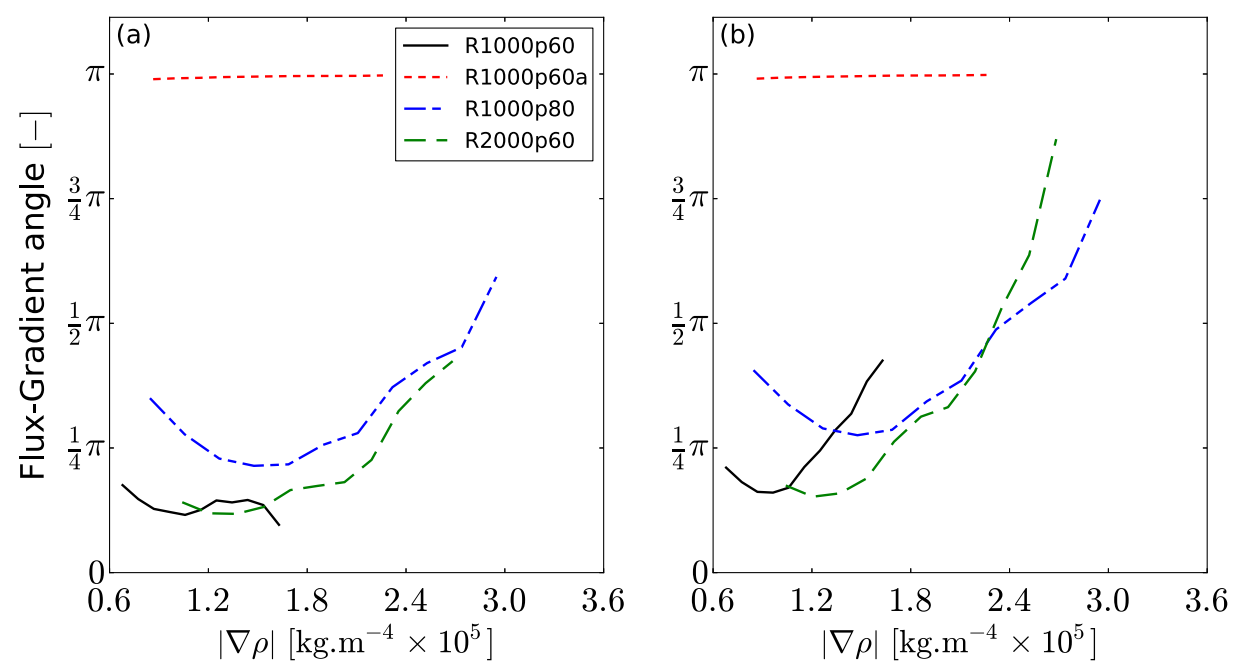

Fig. 9. Conditional averages of the angle between $\mathbf{J}_{\alpha}$ and $\nabla Y_{\alpha}$ on $|\nabla \rho|$ at the respective $t_{p p}^{*}$. (a) $\mathrm{CO}_{2}$, and (b) $\mathrm{H}_{2} \mathrm{O}$.
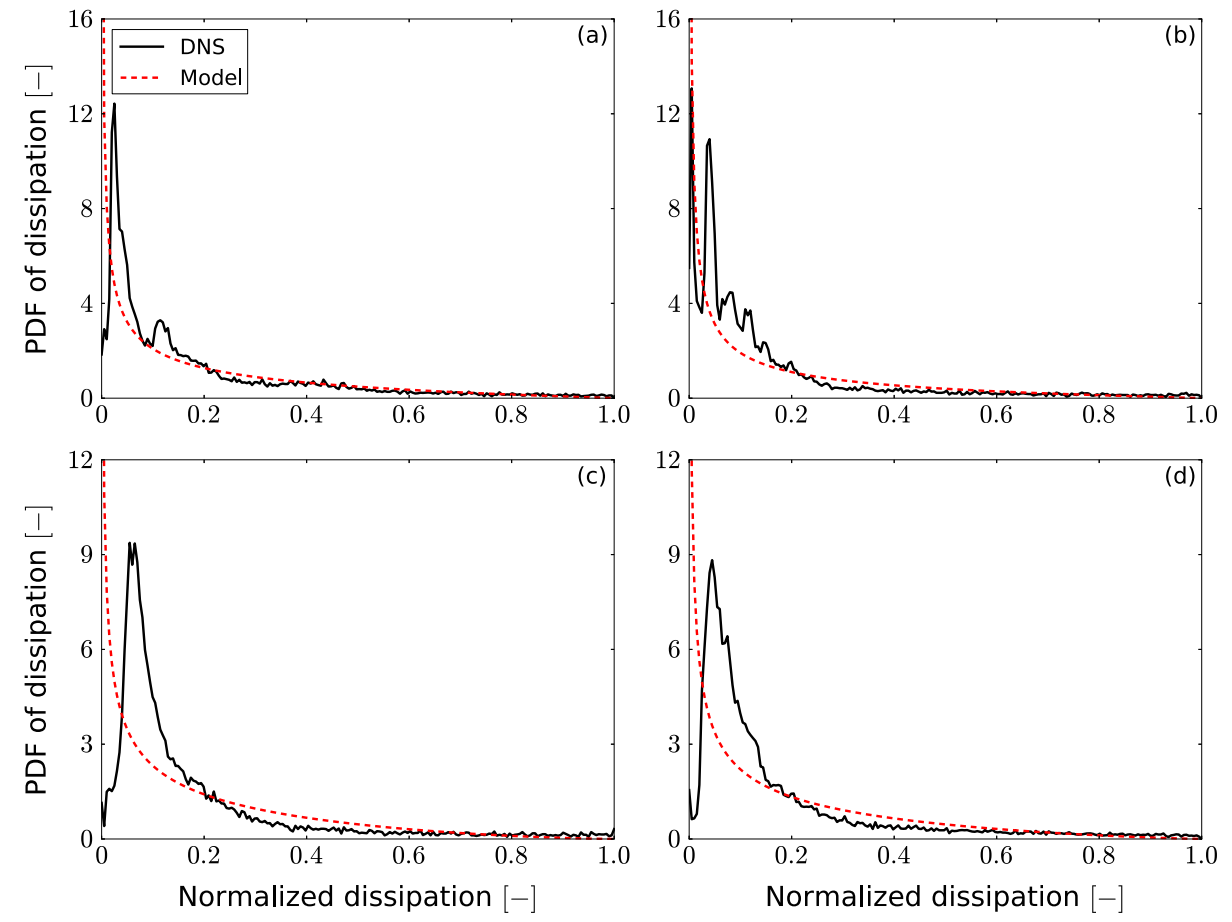

Fig. 10. Dissipation probability density function at $t_{p p}^{*}$ for (a) R1000p60, (b) R2000p60, (c) R1000p80 and (d) R1000p60a. The model is the log normal distribution computed with the exact moments extracted from the DNS. 